Article

\title{
Independent Domination Stable Trees and Unicyclic Graphs
}

\author{
Pu Wu ${ }^{1}$, Huiqin Jiang ${ }^{1}$, Sakineh Nazari-Moghaddam ${ }^{2}{ }^{\circledR}$, Seyed Mahmoud Sheikholeslami ${ }^{2}{ }^{\circledR}$, \\ Zehui Shao ${ }^{1, *(\mathbb{D})}$ and Lutz Volkmann ${ }^{3}$ \\ 1 Institute of Computing Science and Technology, Guangzhou University, Guangzhou 510006, China \\ 2 Department of Mathematics, Azarbaijan Shahid Madani University, Tabriz 5375171379, Iran \\ 3 Lehrstuhl II für Mathematik, RWTH Aachen University, 52056 Aachen, Germany \\ * Correspondence: zshao@gzhu.edu.cn
}

Received: 1 August 2019; Accepted: 2 September 2019; Published: 5 September 2019

check for updates

\begin{abstract}
A set $S \subseteq V(G)$ in a graph $G$ is a dominating set if $S$ dominates all vertices in $G$, where we say a vertex dominates each vertex in its closed neighbourhood. A set is independent if it is pairwise non-adjacent. The minimum cardinality of an independent dominating set on a graph $G$ is called the independent domination number $i(G)$. A graph $G$ is ID-stable if the independent domination number of $G$ is not changed when any vertex is removed. In this paper, we study basic properties of ID-stable graphs and we characterize all ID-stable trees and unicyclic graphs. In addition, we establish bounds on the order of ID-stable trees.
\end{abstract}

Keywords: independent domination; stable graph; tree; unicyclic graph

\section{Introduction}

Throughout this paper, $V(G)$ and edge set $E(G)$ (briefly $V, E$ ) are used to denote the vertex set and edge set of $G$, respectively. For every vertex $v \in V(G)$, the open neighborhood of $v$ is the set $N_{G}(v)=$ $N(v)=\{u \in V(G) \mid u v \in E(G)\}$, and its closed neighborhood is the set $N_{G}[v]=N[v]=N(v) \cup\{v\}$. The degree of a vertex $v \in V$ is $d_{G}(v)=|N(v)|$. A leaf of $G$ is a vertex with degree one, and a support vertex is a vertex adjacent to a leaf. The set of all leaves adjacent to a vertex $v$ is denoted by $L(v)$. For two vertices $u$ and $v$, the distance $d_{G}(u, v)$ from $u$ to $v$ is the number of the edges of a shortest $u v$-path in $G$. The diameter $\operatorname{diam}(G)$ of a graph $G$ is the greatest distance among a pair of vertices of $G$. Assume $T$ is a rooted tree and $v \in V(T)$, let $C(v)$ and $D(v)$ denote the set of children and descendants of $v$, respectively, and $D[v]=D(v) \cup\{v\}$. The maximal subtree at $v$, denoted by $T_{v}$, is the subgraph of $T$ induced by $D[v]$, and is denoted by $T_{v}$. For a graph $G$, let $I(G)$ be the set of vertices with degree 1 . The path and cycle on $n$ vertices are denote by $P_{n}$ and $C_{n}$, respectively.

A set $S \subseteq V$ in a graph $G$ is a dominating set if every vertex of $G$ is either in $S$ or adjacent to a vertex of $S$. The domination number $\gamma(G)$ equals the minimum cardinality of a dominating set in $G$. There are many variants of the dominating set which are studied extensively, such as the independent dominating set [1], total domination [2,3], Roman domination [4,5], semitotal domination [6,7], etc. For a comprehensive treatment of domination in graphs, see the monographs by Haynes, Hedetniemi, and Slater $[8,9]$.

A set is independent if it is pairwise non-adjacent. The minimum cardinality among all independent dominating sets on a graph $G$ is called the independent domination number $i(G)$ of $G$. An $i(G)$-set is an independent dominating set of $G$ of cardinality $i(G)$. This variation of graph domination has been studied extensively in the literature; see for example the books [8,9], and the readers can consult the new survey of Goddard and Henning [1]. 
The removal of a vertex from a graph can increase the independent domination number, decrease the independent domination number, or leave it unchanged. A graph $G$ is independent domination vertex-critical or $i$-vertex-critical if $i(G-v)<i(G)$ for every $v \in V(G)$. The independent domination vertex-critical graphs have been studied by Ao [10] and Edwards [11] and elsewhere [12-14]. Here we focus on the case where the removal of any vertex leave the independent domination number unchanged.

A graph $G$ is independent domination stable (ID-stable) if the independent domination number of $G$ is not changed when any vertex is removed. The domination stable problem consists of characterize graphs whose domination number (a type of domination number, e.g. total domination number, Roman domination number) remains unchanged under removal of any vertex or edge, or addition of any edge [2,15-17].

In this paper, we study basic properties of ID-stable graphs and we characterize all ID-stable trees and unicyclic graphs. In addition, we establish bounds on the order of ID-stable trees.

We make use of the following results in this paper.

Proposition 1 ([1]). For $n \geq 3, i\left(P_{n}\right)=i\left(C_{n}\right)=\left\lceil\frac{n}{3}\right\rceil$.

The next result is an immediate consequence of Proposition 1.

Corollary 1. If $n \geq 3$, then $C_{n}$ is an ID-stable graph if and only if $n \not \equiv 1(\bmod 3)$.

In the next sections, we will use the following notations:

For a graph $G$, let:

$$
W(G)=\{u \in V(G) \mid \text { there exists an } i(G) \text {-set containing } u\}
$$

and:

$$
W^{1,1}(G)=\{(u, v) \mid u, v \in V(G) \text { and there exists an } i(G) \text {-set containing both of } u \text { and } v\} .
$$

\section{Basic Properties}

In this section, we study the basic properties of the ID-stable graph, and we construct new ID-stable graphs from an old one.

Proposition 2. If $G$ is an ID-stable graph, then every support vertex in $G$ is adjacent to exactly one leaf.

Proof. Let $G$ be an ID-stable graph. Suppose, to the contrary, that $G$ has a support vertex $x$ with $|L(x)| \geq 2$, and let $y, z \in L(x)$. If $G$ has an $i(G)$-set $S$ such that $x \notin S$, then $y, z \in S$, and clearly, $S-\{y\}$ is an independent dominating set of $G-y$ yielding $i(G-y)<i(G)$, which is a contradiction. Hence, we assume that every $i(G)$-set contains $x$. Now, consider the graph $G-x$, and let $D$ be an $i(G-x)$-set. Since each vertex in $L(x)$ is isolated in $G-x, D$ contains all vertices in $L(x)$. Clearly, $D$ is an independent dominating set of $G$ such that $x \notin D$. It follows from the assumption that $i(G-x)=|D|>i(G)$, a contradiction again. This completes the proof.

Proposition 3. If $G$ is an ID-stable graph, then $G$ does not have two adjacent support vertices.

Proof. Let $G$ be an ID-stable graph. Suppose, to the contrary, that there exist two adjacent support vertices $x, y$ in $G$. Assume that $L(x)=\left\{x^{\prime}\right\}, L(y)=\left\{y^{\prime}\right\}$, and let $S$ be an $i(G)$-set. Then, $x^{\prime} \in S$ or $y^{\prime} \in S$. Assume, without loss of generality, that $y^{\prime} \in S$. Then, $y \notin S$. If $\left(N_{G}(y)-\left\{y^{\prime}\right\}\right) \cap S \neq \varnothing$, then $S-\left\{y^{\prime}\right\}$ is an independent dominating set of $G-y^{\prime}$, which leads to a contradiction. Hence, 
$\left(N_{G}(y)-\left\{y^{\prime}\right\}\right) \cap S=\varnothing$. In particular, $x \notin S$, and so, $x^{\prime} \in S$. Now, $\left(S-\left\{y^{\prime}, x^{\prime}\right\}\right) \cup\{y\}$ is an independent dominating set of $G-x^{\prime}$, which leads to a contradiction.

The spider $S_{q}$ is the graph obtained from the star $K_{1, q}$ by subdividing its edges once. Clearly, $i\left(S_{q}\right)=q$. Assume that $V\left(S_{q}\right)=\{s\} \cup\left\{a_{i}, b_{i} \mid i=1,2, \ldots, q\right\}$ and $E\left(S_{q}\right)=\left\{s a_{i}, a_{i} b_{i} \mid i=1,2, \ldots, q\right\}$. The vertex $s$ is called the head; the vertices $a_{i}$ are called the knees; and the vertices $b_{i}$ are called the feet of the spider for $1 \leq i \leq q$.

Proposition 4. Let $G$ be a graph and $v \in V(G)$. Let $G^{\prime}$ be the graph obtained from $G$ by adding a spider $S_{q}(q \geq 1)$ and possibly joining the head s to $v$. Then, $i\left(G^{\prime}\right)=i(G)+q$.

Proof. Clearly, any $i(G)$-set can be extended to an independent dominating set of $G^{\prime}$ by adding $a_{1}, \ldots, a_{q}$, and so, $i\left(G^{\prime}\right) \leq i(G)+q$.

Now, we show that $i\left(G^{\prime}\right) \geq i(G)+q$. Let $S$ be an $i\left(G^{\prime}\right)$-set. To dominate $b_{i}$, we must have $\left|S \cap\left\{a_{i}, b_{i}\right\}\right| \geq 1$ for each $i$. If $s \notin S$ or $s v \notin E\left(G^{\prime}\right)$, then the set $S-V\left(S_{q}\right)$ is an independent dominating set of $G$, and this implies that $i\left(G^{\prime}\right) \geq i(G)+q$. Suppose that $s \in S$ and $s v \in E\left(G^{\prime}\right)$. It follows that $\left\{b_{1}, \ldots, b_{q}\right\} \subseteq S$ and $S \cap N_{G}[v]=\varnothing$. Then, the set $\left(S-\left\{s, b_{1}, \ldots, b_{q}\right\}\right) \cup\{v\}$ is an independent dominating set of $G$ yielding $i\left(G^{\prime}\right) \geq i(G)+q$. Thus, $i\left(G^{\prime}\right)=i(G)+q$, and the proof is complete.

Proposition 5. Let $G$ be an ID-stable graph. Then:

1. if $u \in W(G)$ and $G^{\prime}$ is a graph obtained from $G$ by adding a spider $S_{1}$ with head s and an edge us, then $G^{\prime}$ is an ID-stable graph,

2. if $u \in V(G)$ and $G^{\prime}$ is a graph obtained from $G$ by adding a spider $S_{q}(q \geq 2)$ with head s and an edge $u$, then $G^{\prime}$ is an ID-stable graph.

Proof. Our arguments apply equally well to both parts, so we prove them simultaneously. Let $v \in V\left(G^{\prime}\right)$ be an arbitrary vertex. If $v \in V(G)$, then we have $i(G-v)=i(G)$ because $G$ is an ID-stable graph, and by Proposition 4, we have

$$
i\left(G^{\prime}-v\right)=i(G-v)+q=i(G)+q=i\left(G^{\prime}\right) .
$$

Assume that $v \in V\left(S_{q}\right)$. We consider three cases.

Case 1. $v=s$.

Then, clearly, $G^{\prime}-v$ is the union of $G$ with $q K_{2}(q \geq 1)$, and so, $i\left(G^{\prime}-v\right)=i(G)+q$. It follows from Proposition 4 that $i\left(G^{\prime}-v\right)=i\left(G^{\prime}\right)$.

Case 2. $v=a_{i}$ for some $i \in\{1,2, \ldots, q\}$.

Assume, without loss of generality, that $v=a_{q}$. First, we prove (1). Clearly, we have $G^{\prime}-v=$ $(G+u s) \cup K_{1}$. Obviously, any $i(G)$-set containing $u$ can be extended to an independent dominating set of $G^{\prime}-v$ by adding $b_{1}$, and so, $i\left(G^{\prime}-v\right) \leq i(G)+1=i\left(G^{\prime}\right)$. On the other hand, any $i\left(G^{\prime}-v\right)$-set is obviously an independent dominating set of $G^{\prime}$, and so, $i\left(G^{\prime}-v\right) \geq i\left(G^{\prime}\right)$, yielding $i\left(G^{\prime}-v\right)=i\left(G^{\prime}\right)$. Now, we prove (2). Clearly, any $i(G)$-set can be extended to an independent dominating set of $G^{\prime}-v$ by adding $\left\{b_{q}, a_{1}, \ldots, a_{q-1}\right\}$, and so, $i\left(G^{\prime}-v\right) \leq i(G)+q=i\left(G^{\prime}\right)$. Furthermore, any $i\left(G^{\prime}-v\right)$-set is obviously an independent dominating set of $G^{\prime}$, and so, $i\left(G^{\prime}-v\right) \geq i\left(G^{\prime}\right)$. Thus, $i\left(G^{\prime}-v\right)=i\left(G^{\prime}\right)$.

Case 3. $v=b_{i}$ for some $i \in\{1,2, \ldots, q\}$.

Assume, without loss of generality, that $v=b_{1}$. Obviously, any $i(G)$-set can be extended to an independent dominating set of $G^{\prime}-v$ by adding $\left\{a_{1}, \ldots, a_{q}\right\}$, and so, $i\left(G^{\prime}-v\right) \leq i(G)+q=i\left(G^{\prime}\right)$. Now, let $S^{\prime}$ be an $i\left(G^{\prime}-v\right)$-set. If $a_{1} \in S^{\prime}$, then $S^{\prime}$ is obviously an independent dominating set of $G^{\prime}$, and so, $i\left(G^{\prime}-v\right) \geq i\left(G^{\prime}\right)$. Assume that $a_{1} \notin S^{\prime}$. Then, $s \in S^{\prime}$. If $\left(N_{G^{\prime}}(u)-\{s\}\right) \cap S^{\prime} \neq \varnothing$, then $\left(S^{\prime}-\{s\}\right) \cup\left\{a_{1}\right\}$ is an independent dominating set of $G^{\prime}$, and so, $i\left(G^{\prime}-v\right) \geq i\left(G^{\prime}\right)$. Suppose that 
$\left(N_{G^{\prime}}(u)-\{s\}\right) \cap S^{\prime}=\varnothing$. Then, $S^{\prime}-\left\{s, b_{2}, \ldots, b_{q}\right\}$ is an independent dominating set of $G-u$, and since $G$ is an ID-stable graph, we deduce that $i\left(G^{\prime}-v\right)=\left(\left|S^{\prime}\right|-q\right)+q \geq i(G)+q=i\left(G^{\prime}\right)$. Hence, $i\left(G^{\prime}-\right.$ $v)=i\left(G^{\prime}\right)$. Therefore, $G^{\prime}$ is an ID-stable, and the proof is complete.

Let $k_{1}$ and $k_{2}$ be non-negative integers, and let $H_{k_{1}, k_{2}}$ be the graph obtained from the star $K_{1,3}$ centered at $s$ with $V\left(K_{1,3}\right)=\{s, a, b, c\}$ by attaching $k_{1}$ pendent paths $P_{2}$ to $a$ and $k_{2}$ pendent paths $P_{2}$ to $b$ (see, e.g., Figure 1 ). For each $1 \leq i \leq k_{1}$, the vertex set of $i^{\text {th }} P_{2}$ is $\left\{r_{i}, t_{i}\right\}$ with $a r_{i} \in E\left(H_{k_{1}, k_{2}}\right)$, and for each $1 \leq j \leq k_{2}$, the vertex set of $j^{\text {th }} P_{2}$ is $\left\{p_{j}, q_{j}\right\}$ with $b p_{j} \in E\left(H_{k_{1}, k_{2}}\right)$.

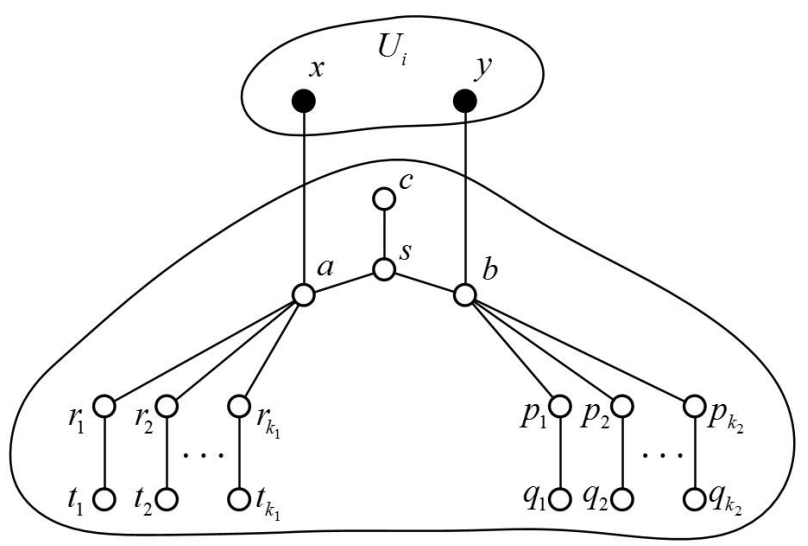

Figure 1. The operations $\mathcal{O}_{3}$, or $\mathcal{O}_{4}$, or $\mathcal{O}_{5}$.

Proposition 6. Let $G$ be a graph and $x, y \in V(G)$ (possibly $x=y$ ). Let $G^{\prime}$ be the graph obtained from $G$ by adding a graph $H_{k_{1}, k_{2}}$ and adding possibly the edges $x a$ or $y b$. Then, $i\left(G^{\prime}\right)=i(G)+k_{1}+k_{2}+1$.

Proof. Clearly, any $i(G)$-set can be extended to an independent dominating set of $G^{\prime}$ by adding $s, r_{1}, \ldots, r_{k_{1}}, p_{1}, \ldots, p_{k_{2}}$, and so, $i\left(G^{\prime}\right) \leq i(G)+k_{1}+k_{2}+1$.

Now, we show that $i\left(G^{\prime}\right) \geq i(G)+k_{1}+k_{2}+1$. Let $S$ be an $i\left(G^{\prime}\right)$-set such that $|S \cap\{a, b\}|$ is as small as possible. To dominate $c, t_{i}\left(1 \leq i \leq k_{1}\right)$ and $q_{j}\left(1 \leq j \leq k_{2}\right)$, we must have $|S \cap\{s, c\}| \geq 1$, $\left|S \cap\left\{r_{i}, t_{i}\right\}\right| \geq 1\left(1 \leq i \leq k_{1}\right)$ and $\left|S \cap\left\{p_{j}, q_{j}\right\}\right| \geq 1\left(1 \leq j \leq k_{2}\right)$. We claim that $|S \cap\{a, b\}|=0$. Suppose, on the contrary, that $|S \cap\{a, b\}| \geq 1$. We consider the following cases.

Case 4. $|S \cap\{a, b\}|=1$.

Assume without loss of generality that $a \in S$ and $b \notin S$. Then, we must have $c \in S$ and $t_{1}, \ldots, t_{k_{1}} \in S$ if $k_{1} \geq 1$. If $x a \notin E\left(G^{\prime}\right)$ or $S \cap N_{G}(x) \neq \varnothing$, then the set $(S-\{a, c\}) \cup\{s\}$ is an independent dominating set of $G^{\prime}$ of size less that $i\left(G^{\prime}\right)$, which is a contradiction. Hence, $x a \in E\left(G^{\prime}\right)$ or $S \cap N_{G}(x)=\varnothing$, but then the set $(S-\{a\}) \cup\{x\}$ is an $i\left(G^{\prime}\right)$-set, which contradicts the choice of $S$.

Case 5. $|S \cap\{a, b\}|=2$.

Then, we must have $c \in S,\left\{t_{1}, \ldots, t_{k_{1}}\right\} \subseteq S$ if $k_{1} \geq 1$ and $\left\{q_{1}, \ldots, q_{k_{2}}\right\} \subseteq S$ if $k_{2} \geq 1$. If $S \cap$ $N_{G}[x] \neq \varnothing$ and $S \cap N_{G}[y] \neq \varnothing$, then $(S-\{a, b, c\}) \cup\{s\}$ is an independent dominating set of $G^{\prime}$ of size $i\left(G^{\prime}\right)-2$, which is a contradiction. Assume without loss of generality that $S \cap N_{G}[x]=\varnothing$. If $x=y$, then $(S-\{a, b, c\}) \cup\{x, s\}$ is an independent dominating set of $G^{\prime}$ of size $i\left(G^{\prime}\right)-1$, a contradiction again. Hence, $x \neq y$. Now, to dominate $x$, we must have $x a \in E\left(G^{\prime}\right)$, but then the set $(S-\{a\}) \cup\{x\}$ is an $i(G)^{\prime}$-set, contradicting the choice of $S$.

Therefore $a, b \notin S$. Now, the set $S \cap V(G)$ is an independent dominating set of $G$, and this implies that $i\left(G^{\prime}\right) \geq i(G)+k_{1}+k_{2}+1$. Thus, $i\left(G^{\prime}\right)=i(G)+k_{1}+k_{2}+1$, and the proof is complete.

Proposition 7. Let G be an ID-stable graph. Then:

(a) if $(x, y) \in W^{1,1}(G)$ and $G^{\prime}$ is a graph obtained from $G$ by adding $H_{0,0}$ and adding the edges $x a, y b$, then $G^{\prime}$ is an ID-stable graph, 
(b) if $x \in W(G), y \in V(G)$, and $G^{\prime}$ is a graph obtained from $G$ by adding $H_{0, k_{2}}\left(k_{2} \geq 1\right)$ and adding the edges $x a, y b$, then $G^{\prime}$ is an ID-stable graph,

(c) if $x, y \in V(G)$ and $G^{\prime}$ is a graph obtained from $G$ by adding $H_{k_{1}, k_{2}}\left(k_{1}, k_{2} \geq 1\right)$ and adding the edges $x a, y b$, then $G^{\prime}$ is an ID-stable graph.

Proof. Let $v$ be a vertex in $G^{\prime}$. If $v \in V(G)$, then by Proposition 6, we have $i\left(G^{\prime}-v\right)=i(G-v)+k_{1}+$ $k_{2}+1$. Since $G$ is an ID-stable graph, we have $i(G-v)=i(G)$, and so, $i\left(G^{\prime}-v\right)=i(G)+k_{1}+k_{2}+1=$ $i\left(G^{\prime}\right)$. Assume that $v \notin V(G)$. We consider the following cases.

Case 6. $v=s$.

Clearly, any $i\left(G^{\prime}-v\right)$-set is an independent dominating set of $G^{\prime}$, and so, $i\left(G^{\prime}-v\right) \geq i\left(G^{\prime}\right)$. In the case (a), any $i(G)$-set containing $x, y$ can be extended to an independent dominating set of $G^{\prime}-v$ by adding $c$, and so, $i\left(G^{\prime}-v\right) \leq i(G)+1=i\left(G^{\prime}\right)$. In the case (b), any $i(G)$-set containing $x$ can be extended to an independent dominating set of $G^{\prime}-v$ by adding $c, p_{1}, \ldots, p_{k_{2}}$, and so, $i\left(G^{\prime}-v\right) \leq$ $i(G)+k_{2}+1=i\left(G^{\prime}\right)$. In the case (c), any $i(G)$-set can be extended to an independent dominating set of $G^{\prime}-v$ by adding $c, p_{1}, \ldots, p_{k_{2}}, r_{1}, \ldots, r_{k_{1}}$, and so, $i\left(G^{\prime}-v\right) \leq i(G)+k_{1}+k_{2}+1=i\left(G^{\prime}\right)$. Thus, $i\left(G^{\prime}-v\right)=i\left(G^{\prime}\right)$.

Case 7. $v=a$ (the case $v=b$ is similar).

It is easy to see that there exists an $i\left(G^{\prime}-v\right)$-set containing $s$. On the other hand, any $i\left(G^{\prime}-v\right)$-set containing $s$ is an independent dominating set of $G^{\prime}$, and so, $i\left(G^{\prime}-v\right) \geq i\left(G^{\prime}\right)$. Using an argument similar to that described in Case 6, we obtain $i\left(G^{\prime}-v\right)=i\left(G^{\prime}\right)$.

Case 8. $v=c$.

Obviously, any $i(G)$-set can be extended to an independent dominating set of $G^{\prime}-v$ by adding the vertices $s, r_{1}, \ldots, r_{k_{1}}$ if $k_{1} \geq 1$ and $p_{1}, \ldots, p_{k_{2}}$ if $k_{2} \geq 1$, and so, $i\left(G^{\prime}-v\right) \leq i(G)+k_{1}+k_{2}+1=i\left(G^{\prime}\right)$. Now, let $S$ be an $i\left(G^{\prime}-v\right)$-set. To dominate $s, t_{i}\left(1 \leq i \leq k_{1}\right)$ and $q_{j}\left(1 \leq j \leq k_{2}\right)$, we must have $|S \cap\{a, b, s\}| \geq 1,\left|S \cap\left\{r_{i}, t_{i}\right\}\right| \geq 1$ for $1 \leq i \leq k_{1}$ and $\left|S \cap\left\{p_{j}, q_{j}\right\}\right| \geq 1$ for $1 \leq j \leq k_{2}$. If $s \in S$, then $S$ is obviously an independent dominating set of $G^{\prime}$, and so, $i\left(G^{\prime}-v\right) \geq i\left(G^{\prime}\right)$. Assume that $s \notin S$. Then, $a \in S$ or $b \in S$. Assume, without loss of generality, that $a \in S$. If $b \notin S$, then $S-V\left(H_{k_{1}, k_{2}}\right)$ is an independent dominating set of $G-x$, and since $G$ is an independent domination stable graph, we have $i\left(G^{\prime}-v\right)=\left(|S|-k_{1}-k_{2}-1\right)+k_{1}+k_{2}+1 \geq i(G)+k_{1}+k_{2}+1=i\left(G^{\prime}\right)$. Let $b \in S$. This implies that $\left\{q_{1}, \ldots, q_{k_{2}}\right\} \subseteq S$ if $k_{2} \geq 1$. If $(N(y)-\{b\}) \cap S \neq \varnothing$, then the set $S-\{b\}$ if $k_{2}=0$, and the set $\left(S-\left\{b, q_{1}, \ldots, q_{k_{2}}\right\}\right) \cup\left\{p_{1}, \ldots, p_{k_{2}}\right\}$ if $k_{2} \geq 1$ is an independent dominating set of $G^{\prime}-v$, which leads to a contradiction. Hence, $(N(y)-\{b\}) \cap S=\varnothing$, and similarly, $(N(x)-\{a\}) \cap S=\varnothing$. Then, $\left(S-V\left(H_{k_{1}, k_{2}}\right)\right) \cup\{y\}$ is an independent dominating set of $G-x$, and since $G$ is an ID-stable graph, we deduce that $i\left(G^{\prime}-v\right)=\left(|S|-k_{1}-k_{2}-1\right)+k_{1}+k_{2}+1 \geq i(G)+k_{1}+k_{2}+1=i\left(G^{\prime}\right)$. Therefore, $i\left(G^{\prime}-v\right)=i\left(G^{\prime}\right)$.

Case 9. $v=r_{i}$ for some $i \in\left\{1,2, \ldots, k_{1}\right\}$ or $v=p_{j}$ for some $j \in\left\{1,2, \ldots, k_{2}\right\}$.

Assume, without loss of generality, that $v=r_{1}$. Obviously, any $i(G)$-set can be extended to an independent dominating set of $G^{\prime}-v$ by adding $s, t_{1}, r_{2}, \ldots, r_{k_{1}}$ and $p_{1}, \ldots, p_{k_{2}}$ if $k_{2} \geq 1$, and so, $i\left(G^{\prime}-v\right) \leq i(G)+k_{1}+k_{2}+1=i\left(G^{\prime}\right)$. On the other hand, any $i\left(G^{\prime}-v\right)$-set is obviously an independent dominating set of $G^{\prime}$, and so, $i\left(G^{\prime}-v\right) \geq i\left(G^{\prime}\right)$. Therefore, $i\left(G^{\prime}-v\right)=i\left(G^{\prime}\right)$.

Case 10. $v=t_{i}$ for some $i \in\left\{1,2, \ldots, k_{1}\right\}$ or $v=q_{j}$ for some $j \in\left\{1,2, \ldots, k_{2}\right\}$.

Assume, without loss of generality that $v=t_{1}$. Clearly, any $i(G)$-set can be extended to an independent dominating set of $G^{\prime}-v$ by adding the vertices $s, r_{1}, \ldots, r_{k_{1}}$ and $p_{1}, \ldots, p_{k_{2}}$ if $k_{2} \geq 1$, and so, $i\left(G^{\prime}-v\right) \leq i(G)+k_{1}+k_{2}+1=i\left(G^{\prime}\right)$. To prove the inverse inequality, let $S$ be an $i\left(G^{\prime}-v\right)$-set. To dominate $c, t_{i}\left(2 \leq i \leq k_{1}\right)$ and $q_{j}\left(1 \leq j \leq k_{2}\right)$, we must have $|S \cap\{c, s\}| \geq 1,\left|S \cap\left\{r_{i}, t_{i}\right\}\right| \geq 1$ for $2 \leq i \leq k_{1}$ and $\left|S \cap\left\{p_{j}, q_{j}\right\}\right| \geq 1$ for $1 \leq j \leq k_{2}$. If $r_{1} \in S$, then $S$ is obviously an independent dominating set of $G^{\prime}$, and so, $i\left(G^{\prime}-v\right) \geq i\left(G^{\prime}\right)$. Assume that $r_{1} \notin S^{\prime}$. It follows that $a \in S$ yielding $c, t_{i} \in S$ for $2 \leq i \leq k_{2}$. If $b \notin S$, then we may assume that $p_{1}, \ldots, p_{k_{2}} \in S$, and clearly, the set $S-V\left(H_{k_{1}, k_{2}}\right)$ is an independent dominating set of $G-x$. Since $G$ is an ID-stable graph, we obtain 
$i\left(G^{\prime}-v\right)=\left(|S|-k_{1}-k_{2}-1\right)+k_{1}+k_{2}+1 \geq i(G)+k_{1}+k_{2}+1=i\left(G^{\prime}\right)$. Let $b \in S$. Then, $q_{1}, \ldots, q_{k_{2}} \in$ $S$ if $k_{2} \geq 1$. It is easy to see that $(N(y)-\{b\}) \cap S=\varnothing$. If $(N(x)-\{a\}) \cap S \neq \varnothing$, then $(S-\{a\}) \cup\left\{r_{1}\right\}$ is an independent dominating set of $G^{\prime}$, and so, $i\left(G^{\prime}-v\right) \geq i\left(G^{\prime}\right)$. Suppose that $(N(x)-\{a\}) \cap S=\varnothing$. Then, $\left(S-V\left(H_{k_{1}, k_{2}}\right)\right) \cup\{y\}$ is an independent dominating set of $G-x$, and since $G$ is an ID-stable graph, we have $i\left(G^{\prime}-v\right)=\left(|S|-k_{1}-k_{2}-1\right)+k_{1}+k_{2}+1 \geq i(G)+k_{1}+k_{2}+1=i\left(G^{\prime}\right)$. Hence, $i\left(G^{\prime}-v\right)=i\left(G^{\prime}\right)$. Thus, $G^{\prime}$ is an ID-stable graph, and the proof is complete.

Let $\mathbb{N}$ be the set of non-negative integers, $n \geq 3$ and $Q \in \mathbb{N}^{n}$ with $Q=\left(q_{1}, q_{2}, \ldots, q_{n}\right)$. Let $D(Q)=$ $\left\{i \mid q_{i}>0\right\}$. For any $i$ with $q_{i}>0$ and $q_{i+1}=0$, if $q_{j}=0$ for $j=i+1, i+2, \ldots, i+k$ and $q_{i+k+1}>0$ where the subscript is taken modulo $n$, we define $H(Q, i)=k$. For example, if $Q=$ $(0,2,0,1,3,0,1,2,0,0)$, then $H(Q, 2)=1, H(Q, 5)=1$ and $H(Q, 8)=3$.

The graph $C(n, Q)$ (resp. $P(n, Q)$ ) is the graph obtained from $C_{n}=\left(v_{1} v_{2} \ldots v_{n}\right)$ (resp. $\left.\quad P_{n}=v_{1} v_{2} \ldots v_{n}\right)$ by attaching $q_{i}$ disjoint pendent paths $P_{2}$ to $v_{i}$. If $q_{i}>0$, then let $H_{v_{i}}=\left\{v_{i+1}, \ldots, v_{i+H(Q, i)}\right\}$, and assume for any $1 \leq j \leq q_{i}$, the vertex set of $j^{\text {th }} P_{2}$ attached to $v_{i}$ is $\left\{v_{i, j, a}, v_{i, j, b}\right\}$ with leaf $v_{i, j, b}$ (see Figure 2).

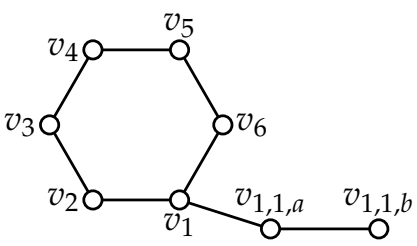

(a)

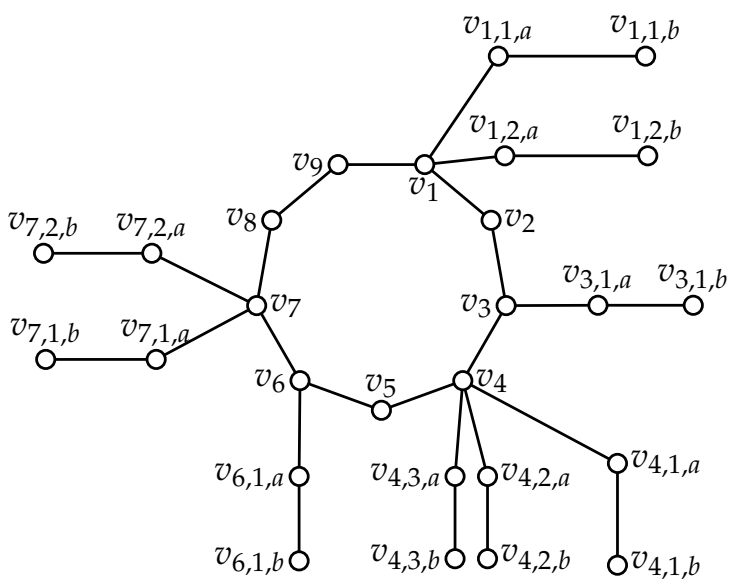

(b)

Figure 2. (a) The graph $C(6,(1,0,0,0,0,0)) ;(\mathbf{b})$ the graph $C(9,(2,0,1,3,0,1,2,0,0))$.

Proposition 8. Let $G$ be a graph, and $x, y \in V(G)$ (possibly $x=y$ ). If $G^{\prime}$ is a graph obtained from $G$ by adding $H=P\left(n,\left(0,0, k_{1}, 0, \ldots, 0, k_{2}, 0,0\right)\right)$, where $n \equiv 0(\bmod 6), k_{1} \geq 0, k_{2} \geq 0$, and adding possibly the edges $x v_{3}$ and $y v_{n-2}$, then $i\left(G^{\prime}\right)=i(G)+k_{1}+k_{2}+\frac{n}{3}$.

Proof. Clearly, any $i(G)$-set can be extended to an independent dominating set of $G^{\prime}$ by adding $v_{3 i-1}\left(1 \leq i \leq \frac{n}{3}\right), v_{3, j, a}\left(1 \leq j \leq k_{1}\right)$ and $v_{n-2, j, a}\left(1 \leq j \leq k_{2}\right)$, and so, $i\left(G^{\prime}\right) \leq i(G)+k_{1}+k_{2}+\frac{n}{3}$.

Now, we show that $i\left(G^{\prime}\right) \geq i(G)+k_{1}+k_{2}+\frac{n}{3}$. Let $S$ be an $i\left(G^{\prime}\right)$-set. To dominate the vertices $v_{1}, v_{n}, v_{3 i-1}\left(2 \leq i \leq \frac{n}{3}-1\right), v_{3, j, b}\left(1 \leq j \leq k_{1}\right)$ and $v_{n-2, j, b}\left(1 \leq j \leq k_{2}\right)$, we must have $\left|S \cap\left\{v_{1}, v_{2}\right\}\right| \geq 1$, $\left|S \cap\left\{v_{n-1}, v_{n}\right\}\right| \geq 1,\left|S \cap\left\{v_{3 i-2}, v_{3 i-1}, v_{3 i}\right\}\right| \geq 1\left(2 \leq i \leq \frac{n}{3}-1\right),\left|S \cap\left\{v_{3, j, a}, v_{3, j, b}\right\}\right| \geq 1(1 \leq j \leq$ $\left.k_{1}\right)$, and $\left|S \cap\left\{v_{n-2, j, a}, v_{n-2, j, b}\right\}\right| \geq 1\left(1 \leq j \leq k_{2}\right)$. We may assume without loss of generality that $\left\{v_{3 i-1} \mid 2 \leq i \leq \frac{n}{3}-1\right\} \subseteq S$. If $v_{3}, v_{n-2} \notin S$, then the set $S \cap V(G)$ is an independent dominating set of $G$, and this implies that $i\left(G^{\prime}\right) \geq i(G)+k_{1}+k_{2}+\frac{n}{3}$. Assume without loss of generality that $v_{3} \in S$. Then, we must have $\left\{v_{3,1, b}, \ldots, v_{3, k_{1}, b}\right\} \subseteq S$ and $S \cap N_{G}\left(v_{3}\right)=\varnothing$. If $v_{3}$ is not adjacent to $x$ or $N_{G}(x) \cap S \neq \varnothing$, then the set $S^{\prime}=\left(S-\left\{v_{1}, v_{2}, v_{3}, v_{3,1}, \ldots, v_{3, k}, b\right\}\right) \cup\left\{v_{2}, v_{3,1, a}, \ldots, v_{3, k_{1}, a}\right\}$ is an independent dominating set of $G^{\prime}$ of size $i\left(G^{\prime}\right)-1$, a contradiction. Hence, $v_{3} x \in E\left(G^{\prime}\right)$, $N_{G}(x) \cap S \neq \varnothing$, and so, $x \notin S$. If $v_{n-2} \notin S$, then the set $(S-V(H)) \cup\{x\}$ is an independent dominating set of $G$, yielding $i\left(G^{\prime}\right) \geq i(G)+k_{1}+k_{2}+\frac{n}{3}$. Assume that $v_{n-2} \in S$. Then, we have $\left\{v_{n-2,1, b}, \ldots, v_{n-2, k_{2}, b}\right\} \subseteq S$ and $S \cap N_{G}\left(v_{n-2}\right)=\varnothing$. Using the above arguments, we have $v_{n-2} y \in$ 
$E\left(G^{\prime}\right), y \notin S$, and $N_{G}(y) \cap S=\varnothing$. If $x=y$ or $x$ and $y$ are adjacent in $G$, then the set $(S-V(H)) \cup$ $\left\{x, v_{2}, v_{5}, \ldots, v_{n-2}, v_{3,1, a}, \ldots, v_{3, k_{1}, a}, v_{n-2,1, a}, \ldots, v_{n-2, k_{2}, a}\right\}$ is an independent dominating set of $G^{\prime}$ of size $i\left(G^{\prime}\right)-1$, which is a contradiction. Hence, $x \neq y$ and $x$ and $y$ are not adjacent in $G$. Now, the set $(S-V(H)) \cup\{x, y\}$ is an independent dominating set of $G$, implying that $i\left(G^{\prime}\right) \geq i(G)+k_{1}+k_{2}+\frac{n}{3}$. Therefore, $i\left(G^{\prime}\right)=i(G)+k_{1}+k_{2}+\frac{n}{3}$, and the proof is complete.

Proposition 9. Let $G$ be an ID-stable graph. If $x, y \in V(G)$ and $G^{\prime}$ is a graph obtained from $G$ by adding $P\left(6,\left(0,0, k_{1}, k_{2}, 0,0\right)\right)$ and adding the edges $x v_{3}, y v_{4}$, then $G^{\prime}$ is an ID-stable graph.

Proof. Let $v$ be a vertex in $G^{\prime}$. If $v \in V(G)$, then by Proposition 8 and the fact that $G$ is an ID-stable graph, we obtain:

$$
i\left(G^{\prime}-v\right)=i(G-v)+k_{1}+k_{2}+2=i(G)+k_{1}+k_{2}+2=i\left(G^{\prime}\right) .
$$

Let $v \notin V(G)$. We consider the following cases.

Case 11. $v \in\left\{v_{2}, v_{5}, v_{3, j, a}, v_{4, k, a} \mid 1 \leq j \leq k_{1}\right.$ and $\left.1 \leq k \leq k_{2}\right\}$.

As in Case 9 in Proposition 7, we have $i\left(G^{\prime}-v\right)=i\left(G^{\prime}\right)$.

Case 12. $v \in\left\{v_{1}, v_{6}, v_{3, j, b}, v_{4, k, b} \mid 1 \leq j \leq k_{1}\right.$ and $1 \leq k \leq k_{2}$.

As in Case 10 in Proposition 7, we have $i\left(G^{\prime}-v\right)=i\left(G^{\prime}\right)$.

Case 13. $v \in\left\{v_{3}, v_{4}\right\}$.

We may assume, without loss of generality, that $v=v_{3}$. Clearly, any $i\left(G^{\prime}-v\right)$-set containing $v_{2}$ is an independent dominating set of $G^{\prime}$, and so, $i\left(G^{\prime}-v\right) \geq i\left(G^{\prime}\right)$. On the other hand, any $i(G)$-set can be extended to an independent dominating set of $G^{\prime}-v$ by adding $\left\{v_{2}, v_{5}, v_{3, j, a}, v_{4, l, a} \mid 1 \leq j \leq\right.$ $k_{1}$ and $\left.1 \leq l \leq k_{2}\right\}$, and Proposition 8 yields $i\left(G^{\prime}-v\right) \leq i(G)+k_{1}+k_{2}+2=i\left(G^{\prime}\right)$.

Proposition 10. Let $Q=\left(q_{1}, q_{2}, \ldots, q_{n}\right) \in \mathbb{N}^{n}$ such that $|D(Q)| \geq 2$ and $H(Q, i) \geq 1$ for each $i \in D(Q)$. If $H(Q, i) \equiv 1(\bmod 3)$ for some $i \in D(Q)$ or $H(i, Q) \equiv 2(\bmod 3)$ for each $i \in D(Q)$, or $H(Q, i) \equiv$ $0(\bmod 3)$ and $H(Q, j) \equiv 0(\bmod 3)$ for some $i, j \in D(Q)$, then the graph $C(n, Q)$ is not an ID-stable graph.

Proof. Suppose, to the contrary, that $G=C(n, Q)$ is an ID-stable graph. If $G$ has an $i(G)$-set $S$ containing $v_{i}$ for some $i \in D(Q)$, then $S-\left\{v_{i, 1, b}\right\}$ is an independent dominating stable set for $G-v_{i, 1, b}$ which leads to a contradiction. Hence, for any $i(G)$-set $S$ and any $i \in D(Q)$, we have $v_{i} \notin S$. Assume that $D(Q)=\left\{i_{1}, i_{2}, \ldots, i_{r}\right\}$. Now, we show that:

$$
i(G)=\sum_{j=1}^{r} q_{i_{j}}+\sum_{j=1}^{r}\left\lceil\frac{H\left(i_{j}, Q\right)}{3}\right\rceil .
$$

For $1 \leq j \leq r$, let $P_{i_{j}}$ be the path $v_{i_{j}+1} v_{i_{j}+2} \ldots v_{i_{j}+H\left(Q, i_{j}\right)}$, and let $S_{j}$ be an $i\left(P_{i_{j}}\right)$-set. Clearly, the set $I=\cup_{j=1}^{r}\left(S_{i_{j}} \cup\left\{v_{i_{j}, k, a} \mid 1 \leq k \leq q_{i_{j}}\right\}\right)$ is an independent dominating set of $G$, and we conclude from Proposition 1 that $i(G) \leq \sum_{j=1}^{r} q_{i_{j}}+\sum_{j=1}^{r}\left\lceil\frac{H\left(i_{j}, Q\right)}{3}\right\rceil$. To prove the inverse inequality, let $S$ be an $i(G)$-set. To dominate the vertices $v_{i_{j}, k, b}$ for $1 \leq j \leq r$ and $1 \leq k \leq q_{i_{j}}$, we must have $\mid S \cap$ $\left\{v_{i_{j}, k, a}, v_{i_{j}, k, b}\right\} \mid \geq 1$, and since $S \cap\left\{v_{i_{1}}, \ldots, v_{i_{r}}\right\}=\varnothing$, we must have $\left|S \cap H_{v_{i_{j}}}\right| \geq\left\lceil\frac{H\left(i_{j}, Q\right)}{3}\right\rceil$ for each $j$, by Proposition 1. This implies that $i(G)=|S| \geq \sum_{j=1}^{r}\left(d_{G}\left(v_{i_{j}}\right)-2\right)+\sum_{j=1}^{r}\left\lceil\frac{H\left(i_{j}, Q\right)}{3}\right\rceil$. Hence, $i(G)=$ $\left.\sum_{j=1}^{r} q_{i_{j}}\right)+\sum_{j=1}^{r}\left\lceil\frac{H\left(i_{j}, Q\right)}{3}\right\rceil$.

If $H\left(Q, i_{j}\right) \equiv 1(\bmod 3)$ for some $i_{j} \in D(Q)$, say $j=1$, then the set $\left(I-\left(S_{i_{1}} \cup\left\{v_{i_{1}, k, a} \mid 1 \leq\right.\right.\right.$ $\left.\left.\left.k \leq q_{i_{1}}\right\}\right)\right) \cup\left(\left\{v_{i_{1}, k, b} \mid 1 \leq k \leq q_{i_{1}}\right\} \cup\left\{v_{i_{1}+3 s} \mid 0 \leq s \leq\left\lceil\frac{q_{i_{1}}}{3}\right\rceil-1\right\}\right)$ when $q_{i_{r}} \equiv 0(\bmod 3)$, the set $\left(I-\left(S_{i_{1}} \cup S_{i_{2}} \cup\left\{v_{i_{1}, k, a} \mid 1 \leq k \leq q_{i_{1}}\right\}\right)\right) \cup\left(\left\{v_{i_{1}, k, b} \mid 1 \leq k \leq q_{i_{1}}\right\} \cup\left\{v_{i_{1}+3 s} \mid 0 \leq s \leq\left\lceil\frac{q_{i_{1}}}{3}\right\rceil\right\} \cup\right.$ $\left.\left\{v_{i_{r}+3 s+1} \mid 0 \leq s \leq\left\lceil\frac{q_{i_{r}}}{3}\right\rceil-1\right\}\right)$ when $q_{i_{r}} \equiv 2(\bmod 3)$, and the set $\left(I-\left(S_{i_{1}} \cup S_{i_{2}} \cup\left\{v_{i_{1}, k, a} \mid 1 \leq k \leq\right.\right.\right.$ 
$\left.\left.\left.q_{i_{1}}\right\}\right)\right) \cup\left(\left\{v_{i_{1}, k, b} \mid 1 \leq k \leq q_{i_{1}}\right\} \cup\left\{v_{i_{1}+3 s} \mid 0 \leq s \leq\left\lceil\frac{q_{i_{1}}}{3}\right\rceil\right\} \cup\left\{v_{i_{r}+q_{i_{r}}-1}, v_{i_{r}+3 s+1} \mid 0 \leq s \leq\left\lceil\frac{q_{i_{r}}}{3}\right\rceil-2\right\}\right)$ when $q_{i_{r}} \equiv 1(\bmod 3)$ is an $i(G)$-set, which is a contradiction. Thus, $H\left(Q, i_{j}\right) \not \equiv 1(\bmod 3)$ for each $i_{j} \in D(Q)$.

Suppose $H(i, Q) \equiv 2(\bmod 3)$ for each $i \in D(Q)$. Then, clearly, $n=\sum_{j=1}^{r}\left\lceil\frac{H\left(i_{j}, Q\right)}{3}\right\rceil$, and the set $\left.\left\{v_{i_{1}+3 s} \mid 0 \leq i \leq\left\lceil\frac{n}{3}\right\rceil-1\right\} \cup\left\{v_{i_{j}, k, b} \mid 1 \leq k \leq q_{i_{j}}\right\}\right)$ is an independent dominating set of $G$, which leads to a contradiction again.

Finally let, without loss of generality, $H\left(Q, i_{1}\right) \equiv 0(\bmod 3)$ and $H\left(Q, i_{\ell}\right) \equiv 0(\bmod 3)$ for some $i_{1}, i_{\ell} \in D(Q)$ and $H\left(Q, i_{j}\right) \equiv 2(\bmod 3)$ for each $i_{j} \in D(Q)-\left\{i_{j}, i_{\ell}\right\}$. If $|D(Q)|=2$, then it is not hard to see that $i\left(G-v_{i_{1}+2}\right)>i(G)$, which is a contradiction. Assume that $|D(Q)| \geq 3$. By symmetry, we may assume that $\ell \geq 3$. Let $G^{\prime}=G-v_{i_{\ell}+2}$, and let $S^{\prime}$ be an $i\left(G^{\prime}\right)$-set such that $\left|S^{\prime} \cap\left\{v_{i_{1}}, \ldots, v_{i_{r}}\right\}\right|$ is as large as possible. Since $G$ is an independent domination stable graph, we have $i(G)=i\left(G^{\prime}\right)$. It is not hard to see that the set:

$$
D_{1}=\left(\left\{v_{i_{2}}, \ldots, v_{i_{\ell}}\right\} \cup\left(\bigcup_{j=2}^{\ell-1}\left\{v_{i_{j}+3 k} \mid 1 \leq k \leq\left\lfloor\frac{H\left(i_{j}, Q\right)-1}{3}\right\rfloor\right\}\right)\right)
$$

is a subset of $S^{\prime}$. It follows that $D_{2}=\cup_{j=2}^{\ell-1}\left\{v_{i_{j}, k, b} \mid 1 \leq k \leq q_{i_{j}}\right\} \subseteq S^{\prime}$. We may also assume that $\left\{v_{i_{1}+3 s-1} \mid 1 \leq s \leq\left\lfloor\frac{H\left(i_{1}, Q\right)}{3}\right\rfloor\right\} \subseteq S^{\prime}$. Let $D_{3}=\cup_{j=2}^{\ell}\left\{v_{i_{j}, k, a} \mid 1 \leq k \leq q_{i_{j}}\right\}$. Clearly, the set:

$$
\left(S^{\prime}-\left(D_{1} \cup D_{2}\right)\right) \cup\left\{v_{i_{\ell}}\right\} \cup D_{3} \cup\left(\bigcup_{j=2}^{\ell}\left\{v_{i_{j}+3 k+1} \mid 0 \leq k \leq\left\lfloor\frac{H\left(i_{j}, Q\right)-1}{3}\right\rfloor\right\}\right)
$$

is an independent dominating set of $G$ of cardinality $\left|S^{\prime}\right|=i(G)$ containing $v_{i_{\ell}}$, which is a contradiction. This completes the proof.

\section{Independent Domination Stable Trees}

In this section, we give a constructive characterization of all ID-stable trees.

In order to present our constructive characterization, we define a family of trees as follows. Let $\mathcal{T}$ be the family of trees $T$ that can be obtained from a sequence $T_{1}, T_{2}, \ldots, T_{k}$ of trees for some $k \geq 1$, where $T_{1}$ is $P_{2}$ and $T=T_{k}$. If $k \geq 2, T_{i+1}$ can be obtained from $T_{i}$ by one of the following operations.

Operation $\mathcal{T}_{1}$ : If $u \in W\left(T_{i}\right)$, then $\mathcal{T}_{1}$ adds a spider $S_{1}$ with head $s$ and an edge $u s$ to obtain $T_{i+1}$ (see Figure 3).

Operation $\mathcal{T}_{2}$ : If $u \in V\left(T_{i}\right)$, then $\mathcal{T}_{2}$ adds a spider $S_{q}(q \geq 2)$ with head $s$ and an edge $u s$ to obtain $T_{i+1}$ (see Figure 3).

Theorem 1. If $T \in \mathcal{T}$, then $T$ is an ID-stable tree.

Proof. If $T$ is $P_{2}$, then obviously $T$ is an ID-stable tree. Suppose now that $T \in \mathcal{T}$. Then there exists a sequence of trees $T_{1}, T_{2}, \ldots, T_{k}(k \geq 1)$ such that $T_{1}$ is $P_{2}$, and if $k \geq 2$, then $T_{i+1}$ can be obtained from $T_{i}$ by one of the Operations $\mathcal{T}_{1}$ or $\mathcal{T}_{2}$. We proceed by induction on the number of operations used to construct $T$. If $k=1$, the result is trivial. Assume the result holds for each tree $T \in \mathcal{T}$ which can be obtained from a sequence of operations of length $k-1$ and let $T^{\prime}=T_{k-1}$. By the induction hypothesis, $T^{\prime}$ is an ID-stable tree. Since $T=T_{k}$ is obtained by one of the Operations $\mathcal{T}_{1}$ or $\mathcal{T}_{2}$ from $T^{\prime}$, we conclude from the Proposition 5 that $T$ is an ID-stable tree.

Next, we characterize all ID-stable trees.

Theorem 2. Let $T$ be a tree of order $n \geq 2$. Then, $T$ is an ID-stable tree if and only if $T \in \mathcal{T}$. 


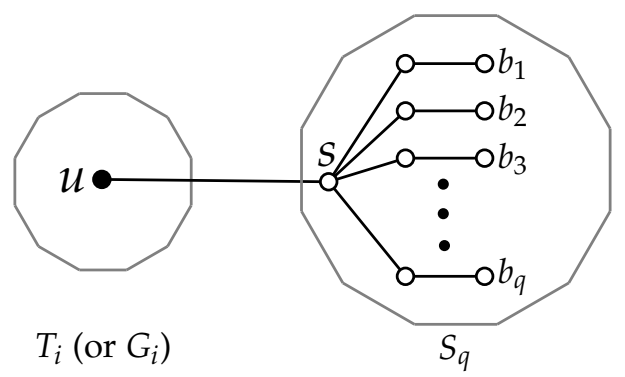

Figure 3. The operations: $\mathcal{T}_{1}$ or $\mathcal{T}_{2}$.

Proof. According to Theorem 1, we need only to prove necessity. Let $T$ be an ID-stable tree of order $n \geq 2$. The proof is by induction on $n$. If $n=2$, then $T=P_{2} \in \mathcal{T}$. Let $n \geq 3$, and let the statement hold for all ID-stable trees of order less than $n$. Assume that $T$ is an ID-stable tree of order $n$. By Propositions 2 and 3 , we deduce that $\operatorname{diam}(T) \geq 4$. Let $v_{1} v_{2} \ldots v_{k}(k \geq 5)$ be a diametrical path in $T$ and root $T$ at $v_{k}$. By Proposition 2, any support vertex adjacent to $v_{3}$ has degree two. In particular $d_{T}\left(v_{2}\right)=2$. By Proposition $3, v_{3}$ is not a support vertex, and so, $T_{v_{3}}=S_{d_{T}\left(v_{3}\right)-1}$. Let $T^{\prime}=T-T_{v_{3}}$. Since $T$ is an ID-stable tree, we deduce from Proposition 4 that for any vertex $v \in V\left(T^{\prime}\right)$,

$$
i\left(T^{\prime}-v\right)+d_{T}\left(v_{3}\right)-1=i(T-v)=i(T)=i\left(T^{\prime}\right)+d_{T}\left(v_{3}\right)-1
$$

and this implies that $i\left(T^{\prime}-v\right)=i\left(T^{\prime}\right)$. Hence, $T^{\prime}$ is an ID-stable tree. It follows from the induction hypothesis that $T^{\prime} \in \mathcal{T}$. If $d_{T}\left(v_{3}\right) \geq 3$, then $T \in \mathcal{T}$ since $T$ can be obtained from $T^{\prime}$ by operation $\mathcal{T}_{2}$.

Assume that $d_{T}\left(v_{3}\right)=2$. By Proposition 4 , we have $i\left(T^{\prime}\right)+1=i(T)$. Since $T$ is an ID-stable tree, we have $i\left(T-v_{2}\right)=i(T)$. Let $S$ be an $i\left(T-v_{2}\right)$-set. Clearly, $v_{1} \in S$. If $v_{3} \in S$, then $S-\left\{v_{1}\right\}$ is an independent dominating set of $T-v_{1}$, which is a contradiction. Hence, $v_{3} \notin S$, and this implies that $v_{4} \in S$. Now, $S-\left\{v_{1}\right\}$ is an independent dominating set of $T^{\prime}$, and we deduce from $i\left(T^{\prime}\right)+1=i(T)$ that $S-\left\{v_{1}\right\}$ is an $i\left(T^{\prime}\right)$-set. Thus, $v_{4} \in W\left(T^{\prime}\right)$. Now, $T$ can be obtained from $T^{\prime}$ by operation $\mathcal{T}_{1}$, and so, $T \in \mathcal{T}$. This completes the proof.

\section{Independent Domination Stable Unicyclic Graphs}

In this section, we give a constructive characterization of all ID-stable unicyclic graphs. We start with introducing the following families of graphs.

- $\mathcal{J}_{1}=\left\{C_{n} \mid n \geq 3\right.$ and $n \not \equiv 1(\bmod$ three $\left.)\right\}$.

- $\mathcal{J}_{2}=\left\{C\left(3 k+1,\left(q_{1}, 0,0, \ldots, 0\right)\right) \mid k \geq 1\right.$ and $\left.q_{1} \geq 2\right\}$.

- $\mathcal{J}_{3}$ is the family of graphs $C(n, Q)$ where $Q=\left(q_{1}, \ldots, q_{n}\right) \in \mathbb{N}^{n}$ satisfies (i) $D(Q) \geq 2$, (ii) $H(Q, i) \not \equiv$ $1(\bmod 3)$ for each $i \in D(Q)$, and (iii) $H(Q, i) \equiv 0(\bmod 3)$ for exactly one $i \in D(Q)$.

- $\mathcal{J}_{4}$ is the family of graphs obtained from $P\left(6,\left(0,0, k_{1}, k_{2}, 0,0\right)\right)\left(k_{1} \geq 0, k_{2} \geq 0\right)$ by adding a new vertex $w$, joining $w$ to $v_{3}, v_{4}$, and adding a pendant edge at $w$ (see, e.g., the graph of the second column and the fifth row in Figure A2 (Appendix A)).

- $\mathcal{J}=\mathcal{J}_{1} \cup \mathcal{J}_{2} \cup \mathcal{J}_{3} \cup \mathcal{J}_{4} \cup \mathcal{T}$.

Next, we show that each graph in $\mathcal{J}$ is an ID-stable graph. By Corollary 1 and Theorem 1 , any graph in the family $\mathcal{T} \cup \mathcal{J}_{1}$ is an independent domination stable graph.

Proposition 11. If $G \in \mathcal{J}_{2}$, then $G$ is an ID-stable graph.

Proof. Let $G \in \mathcal{J}_{2}$. First, we show that $i(G)=k+q_{1}$. Clearly, the set $\left\{v_{3 i} \mid 1 \leq i \leq k\right\} \cup\left\{v_{1, j, a} \mid 1 \leq\right.$ $\left.j \leq q_{1}\right\}$ is an independent dominating set of $G$ yielding $i(G) \leq k+q_{1}$. To prove the inverse inequality, let $S$ be an $i(G)$-set. To dominate $v_{1, j, b}$, we must have $\left|S \cap\left\{v_{1, j, a}, v_{1, j, b}\right\}\right| \geq 1$ for each $j \in\left\{1, \ldots, q_{1}\right\}$. On the other hand, to dominate the vertices $v_{3 i}(1 \leq i \leq k)$, we must have $\left|S \cap\left\{v_{3 i-1}, v_{3 i}, v_{3 i+1}\right\}\right| \geq 1$ for each $i \in\{1, \ldots, k\}$, and this implies that $i(G) \geq k+q_{1}$. Hence, $i(G)=k+q_{1}$. 
Next we show that $G$ is an ID-stable graph. Let $v \in G$. If $v=v_{1}$, then $G=P_{3 k} \cup q_{1} K_{2}$, and by Proposition 1, we have $i(G-v)=i\left(P_{3 k}\right)+i\left(q_{1} K_{2}\right)=k+q_{1}=i(G)$. If $v=v_{1, j, a}$ for some $1 \leq j \leq q_{1}$, then $G=K_{1} \cup C\left(3 k+1,\left(q_{1}-1,0,0, \ldots, 0\right)\right)$, and as above, we have $i(G-v)=k+q_{1}=i(G)$. Suppose that $v=v_{1, j, b}$ for some $1 \leq j \leq q_{1}$, say $j=1$. Clearly, the set $\left\{v_{3 i} \mid 1 \leq i \leq k\right\} \cup\left\{v_{1, j, a} \mid 1 \leq j \leq q_{1}\right\}$ is an independent dominating set of $G$ yielding $i(G-v) \leq k+q_{1}=i(G)$. To prove $i(G-v) \geq k+q_{1}$, let $S^{\prime}$ be an $i(G-v)$-set. To dominate $v_{1, j, a}$, we must have $\left|S^{\prime} \cap\left\{v_{1, j, a}, v_{1}\right\}\right| \geq 1$, and to dominate the vertex $v_{1, j, b}$, we must have $\left|S^{\prime} \cap\left\{v_{1, j, a}, v_{1, j, b}\right\}\right| \geq 1$ for each $j \in\left\{2, \ldots, q_{1}\right\}$. On the other hand, to dominate the vertices in $V\left(C_{n}\right)-\left\{v_{1}, v_{2}, v_{3}\right\}, S^{\prime}$ must contain at least $k-1$ vertices in $\left\{v_{2}, \ldots, v_{n}\right\}$, and so, $i(G-v) \geq k+q_{1}$. Hence, $i(G-v)=i(G)$ in this case. Let now $v=v_{i}(i \neq 1)$. Clearly, any $i\left(P_{3 k}\right)$-set of $P_{3 k}=v_{i-1} \ldots v_{1} v_{n} \ldots v_{i+1}$ can be extended to an independent dominating set of $G-v$ by adding $v_{1, j, a}$ for $j=1,2, \ldots, q_{1}$, and so, $i(G-v) \leq i\left(P_{3 k}\right)+q_{1}=k+q_{1}=i(G)$. On the other hand, if $S$ is an $i(G-v)$-set, then to dominate the vertices in $\left\{v_{1, j, b} \mid 1 \leq j \leq q_{1}\right\}$, we must have $\left|S \cap\left\{v_{1, j, a}, v_{1, j, b} \mid 1 \leq j \leq q_{1}\right\}\right| \geq q_{1}$, and to dominate the vertices in $V\left(C_{n}\right)-\left\{v_{i}, v_{1}\right\}$, we must have $\left|S \cap\left(V\left(C_{n}\right)-\left\{v_{i}\right\}\right)\right| \geq k$. Thus, $i(G-v)=|S| \geq k+q_{1}=i(G)$, and hence, $G$ is an ID-stable graph.

Theorem 3. Let $G=C(n, Q)$ where $n \geq 3$ and $D(Q)=1$. Then, $G$ is an ID-stable graph if and only if $G \in \mathcal{J}_{2}$.

Proof. According to Proposition 11, we only need to prove necessity. Let $G$ be an independent domination stable graph. Assume, without loss of generality, that $Q=\left(q_{1}, 0 \ldots, 0\right)$ where $q_{1} \geq 1$. As Proposition 11, we can see that $i(G)=\left\lceil\frac{n-1}{3}\right\rceil+q_{1}$. If $n \not \equiv 1(\bmod 3)$, then the set $\left\{v_{3 i+1} \mid 0 \leq\right.$ $\left.i \leq\left\lceil\frac{n-1}{3}\right\rceil-1\right\} \cup\left\{v_{1, j, a} \mid 2 \leq j \leq q_{1}\right\}$ is an independent dominating set of $G-v_{1,1, b}$ of size $i(G)-1$, which is a contradiction. Assume that $n \equiv 1(\bmod 3)$. If $q_{1}=1$, then clearly $G-v_{1,1, a}=K_{1} \cup C_{n}$, and by Proposition 1, we have $i(G)=i\left(C_{n}\right)+1=\left\lceil\frac{n-1}{3}\right\rceil+2$, which is a contradiction. Therefore, $q_{1} \geq 2$, and so, $G \in \mathcal{J}_{2}$.

Proposition 12. If $G \in \mathcal{J}_{3}$, then $G$ is an independent domination stable graph.

Proof. Let $G=C(n, Q) \in \mathcal{J}_{3}$, and let $\omega=\sum_{j=1}^{r} q_{i_{j}}+\sum_{j=1}^{r}\left\lceil\frac{H\left(Q, i_{j}\right)}{3}\right\rceil$. Assume that $D(Q)=\left\{i_{1}, \ldots, i_{r}\right\}$, and suppose, without loss of generality, that $H\left(Q, i_{1}\right) \equiv 0(\bmod 3)$. Let $S^{\prime}=\cup_{j=1}^{r}\left\{v_{i_{j}, s, a} \mid 1 \leq s \leq q_{i_{j}}\right\}$, $S^{\prime \prime}=\cup_{j=2}^{r}\left\{v_{i_{j}, s, b} \mid 1 \leq s \leq q_{i_{j}}\right\}, S_{j}^{p}=\left\{v_{i_{j}+3 k+p} \mid 0 \leq k \leq\left\lceil\frac{H\left(Q, i_{j}\right)}{3}\right\rceil-1\right\}$ for $j \in\{1, \ldots, r\}$ and $p \in\{1,2,3\}$.

First, we show that $i(G)=\omega$. Clearly, the set $S=\left(\cup_{j=1}^{r} S_{j}^{2}\right) \cup S^{\prime}$ is an $i(G)$-set, and so, $i(G) \leq \omega$. To prove the inverse inequality, let $T$ be an $i(G)$-set. To dominate the vertices $v_{i, s, b}$, we must have $\left|T \cap\left\{v_{i_{j}, s, a}, v_{i_{j}, s, b}\right\}\right| \geq 1$ for each $1 \leq j \leq r$ and $1 \leq s \leq q_{i_{j}}$. Now, to dominate the vertices $v_{i_{1}+3 k+2}$, we must have $\left|T \cap\left\{v_{i_{1}+3 k+1}, v_{i_{1}+3 k+2}, v_{i_{1}+3 k+3}\right\}\right| \geq 1$ for $0 \leq k \leq\left\lceil\frac{H\left(Q, i_{j}\right)}{3}\right\rceil-1$, and to dominate the vertices $v_{i_{j}+2}, \ldots, v_{i_{j+1}-1}$, we must have $\left|T \cap\left\{v_{i_{j}+1}, v_{i_{j}+2}, \ldots, v_{i_{j+1}-1}, v_{i_{j+1}}\right\}\right| \geq 1$ for each $j \in\{2, \ldots, r\}$ yielding $i(G) \geq \omega$. Thus, $i(G)=\omega$ as desired.

Now, we show that $G$ is an independent domination stable graph. Let $v \in V(G)$. Consider the following cases.

Case 14. $v \in S^{\prime}$.

Clearly, any $i(G-v)$-set is an independent dominating set of $G$, and so, $i(G-v) \geq i(G)$. On the other hand, $\left(\cup_{j=1}^{r} S_{j}^{2}\right) \cup S^{\prime \prime} \cup\left\{v_{i_{1}, s, b} \mid 1 \leq s \leq q_{i_{1}}\right\}$ is an independent dominating set of $G-v$, and hence, $i(G) \geq i(G-v)$. Thus, $i(G)=i(G-v)$ in this case.

Case 15. $v \in\left\{v_{i_{1}}, \ldots, v_{i_{r}}\right\}$.

Suppose, without loss of generality, that $v=v_{i_{1}}$. Obviously, $S$ is an independent dominating set of $G-v$, and hence, $i(G) \geq i(G-v)$. Let $D$ be an $i(G-v)$-set such that $\left|D \cap\left\{v_{i_{1}, j, a} \mid 1 \leq j \leq q_{1}\right\}\right|$ is 
as large as possible. Then, $\left\{v_{i_{1}, j, a} \mid 1 \leq j \leq q_{1}\right\} \subseteq D$. As above, we can see that $|D|=i(G-v) \geq i(G)$. Therefore, $i(G)=i(G-v)$ in this case.

Case 16. $v \in S^{\prime \prime}$.

Assume, without loss of generality, that $v=v_{i_{1}, 1, b}$. Clearly, $S$ is an independent dominating set of $G-v$, and hence, $i(G) \geq i(G-v)$. To prove the inverse inequality, let $T$ be a $i(G-v)$-set. As above, we have $\left|T \cap\left\{v_{i_{1}, s, a}, v_{i_{1}, s, b}\right\}\right| \geq 1$ for $2 \leq s \leq q_{i_{1}}$, and $\left|T \cap\left\{v_{i_{j}, s, a}, v_{i_{j}, s, b}\right\}\right| \geq 1$ for each $2 \leq j \leq r$ and $1 \leq s \leq q_{i_{j}}$. Furthermore, we must have $\left|T \cap\left\{v_{i_{1}}, v_{i_{1}, 1, a}\right\}\right| \geq 1$. Now, to dominate the vertices $v_{i_{1}+2}, \ldots, v_{i_{2}-1}$, we must have $\left|T \cap\left\{v_{i_{1}+1}, \ldots, v_{i_{2}-1}\right\}\right| \geq \frac{H\left(Q, i_{1}\right)}{3}$, and to dominate the vertices $v_{i_{r}+1}, \ldots, v_{i_{1}-2}$, we must have $\left|T \cap\left\{v_{i_{r}}, v_{i_{r}+1}, \ldots, v_{i_{1}-2}, v_{i_{1}-1}\right\}\right| \geq \frac{H\left(Q, i_{r}\right)}{3}$. Repeating this process, we must have $\left|T \cap\left\{v_{i_{i}}, v_{i_{j}+1}, \ldots, v_{i_{j+1}-2}, v_{i_{j+1}-1}\right\}\right| \geq \frac{H\left(Q, i_{j}\right)}{3}$ for each $2 \leq j \leq r-1$. It follows that $|T| \geq i(G)$, and so, $i(G)=i(G-v)$.

Case 17. $v \in S_{1}^{1}$ (the case $v \in S_{1}^{3}$ is similar).

Assume that $v=v_{i_{1}+3 k+1}$. Clearly, the set $\left(\cup_{j=2}^{r} S_{j}^{3}\right) \cup\left\{v_{i_{1}+3 t+2} \mid 0 \leq t \leq k-1\right\} \cup\left\{v_{i_{1}+3 t} \mid k+1 \leq\right.$ $\left.t \leq\left\lceil\frac{H\left(Q, i_{1}\right)-3 k-1}{3}\right\rceil\right\}$ is an independent dominating set of $G-v$ of size $i(G)$, and so, $i(G) \geq i(G-v)$. To prove the inverse inequality, let $T$ be an $i(G-v)$-set. As above, we have $\left|T \cap\left\{v_{i_{j}, s, a}, v_{i_{j}, s, b}\right\}\right| \geq 1$ for each $1 \leq j \leq r$ and $1 \leq s \leq q_{i_{j}}$, and $\left|T \cap\left\{v_{i_{j}+1}, \ldots, v_{i_{j+1}-1}, v_{i_{j+1}}\right\}\right| \geq \frac{H\left(Q, i_{j}\right)}{3}$ for $2 \leq j \leq r$. Now to dominate the vertices $v_{i_{1}+j}(1 \leq j \leq 3 k)$, we must have $\left|T \cap\left\{v_{i_{1}+1}, \ldots, v_{i_{1}+3 k}\right\}\right| \geq k$, and to dominate the vertices $v_{i_{1}+3 k+2}, \ldots, v_{i_{2}-1}$, we must have $\left|T \cap\left\{v_{i_{2}+3 k+2}, \ldots, v_{i_{2}-1}, v_{i_{2}}\right\}\right| \geq \frac{H\left(Q, i_{1}\right)}{3}-k$. This implies that $|T| \geq i(G)$, and so, $i(G)=i(G-v)$.

Case 18. $v \in S_{1}^{2}$. Assume that $v=v_{i_{1}+3 k+2}$. Clearly, the set $\left(\cup_{j=2}^{r} S_{j}^{3}\right) \cup\left\{v_{i_{1}+3 t+3} \mid 0 \leq t \leq\right.$ $k-1\} \cup\left\{v_{i_{1}+3 t+1} \mid k+1 \leq t \leq\left\lceil\frac{H\left(Q, i_{1}\right)-3 k-2}{3}\right\rceil\right\}$ is an independent dominating set of $G-v$ of size $i(G)$, and so, $i(G) \geq i(G-v)$. To prove the inverse inequality, let $T$ be an $i(G-v)$-set. As above, we have $\left|T \cap\left\{v_{i_{j}, s, a}, v_{i_{j}, s, b}\right\}\right| \geq 1$ for each $1 \leq j \leq r$ and $1 \leq s \leq q_{i_{j}}$ and $\left|T \cap\left\{v_{i_{j}+2}, \ldots, v_{i_{j+1}-1}, v_{i_{j+1}}\right\}\right| \geq$ $\frac{H\left(Q, i_{j}\right)}{3}$ for $2 \leq j \leq r$. If $k=0$, then to dominate the vertices $v_{i_{1}+3}, \ldots, v_{i_{2}-1}$, we must have $\left|T \cap\left\{v_{i_{2}+3}, \ldots, v_{i_{2}-1}, v_{i_{2}}\right\}\right| \geq \frac{H\left(Q, i_{1}\right)}{3}$ yielding $|T| \geq i(G)$. If $k \geq 1$, then to dominate the vertices $v_{i_{1}+1}, \ldots, v_{i_{1}+3 k+1}$, we must have $\left|T \cap\left\{v_{i_{1}+2}, \ldots, v_{i_{1}+3 k+1}\right\}\right| \geq k$, and to dominate the vertices $v_{i_{1}+3 k+2}, \ldots, v_{i_{2}-1}$, we must have $\left|T \cap\left\{v_{i_{2}+3 k+2}, \ldots, v_{i_{2}-1}, v_{i_{2}}\right\}\right| \geq \frac{H\left(Q, i_{1}\right)}{3}-k$, so $|T| \geq i(G)$. Therefore, $i(G)=i(G-v)$.

Case 19. $v \in \bigcup_{j=2}^{r} S_{j}^{1}$ (the case $v \in \bigcup_{j=2}^{r} S_{j}^{3}$ is similar).

Suppose, without loss of generality, that $v=v_{i_{2}+3 k+1}$. Clearly, the set $S_{1}^{2} \cup\left(\cup_{j=3}^{r} S_{j}^{2}\right) \cup\left\{v_{i_{2}+3 t+2} \mid\right.$ $0 \leq t \leq k-1\} \cup\left\{v_{i_{3}}, v_{i_{2}+3 t} \mid k+1 \leq t \leq\left\lceil\frac{H\left(Q, i_{2}\right)-3 k-1}{3}\right\rceil\right\}$ is an independent dominating set of $G-v$ of size $i(G)$, and so, $i(G) \geq i(G-v)$. Now, we show that $i(G-v) \geq i(G)$. Let $T$ be a $i(G-v)$-set. As above, we have $\left|T \cap\left\{v_{i_{j}, s, a}, v_{i_{j}, s, b}\right\}\right| \geq 1$ for each $1 \leq j \leq r$ and $1 \leq s \leq q_{i_{j}}$, and $\left|T \cap\left\{v_{i_{1}+1}, v_{i_{1}+2}, \ldots, v_{i_{2}-1}\right\}\right| \geq \frac{H\left(Q, i_{1}\right)}{3}$. Furthermore, to dominate the vertices $v_{i_{j}+2}, \ldots, v_{i_{j+1}-1}$, we must have $\left|T \cap\left\{v_{i_{j}+2}, \ldots, v_{i_{j+1}-1}, v_{i_{j+1}}\right\}\right| \geq \frac{H\left(Q, i_{j}\right)}{3}$ for $3 \leq j \leq r$. Now, to dominate the vertices $v_{i_{2}+1}, \ldots, v_{i_{2}+3 k}$, we must have $\left|T \cap\left\{v_{i_{2}+1}, \ldots, v_{i_{2}+3 k}\right\}\right| \geq k$, and to dominate the vertices $v_{i_{2}+3 k+2}, \ldots, v_{i_{3}-1}$, we must have $\left|T \cap\left\{v_{i_{2}+3 k+2}, \ldots, v_{i_{3}-1}, v_{i_{3}}\right\}\right| \geq \frac{H\left(Q, i_{2}\right)}{3}-k$. This implies that $|T| \geq i(G)$, yielding $i(G)=i(G-v)$.

Case 20. $v \in \bigcup_{j=2}^{r} S_{j}^{2}$.

Suppose, without loss of generality, that $v=v_{i_{2}+3 k+2}$. Clearly, the set $S_{1}^{2} \cup\left(\cup_{j=3}^{r} S_{j}^{2}\right) \cup\left\{v_{i_{2}+3 t} \mid\right.$ $0 \leq t \leq k\} \cup\left\{v_{i_{2}+3 t+1} \mid k+1 \leq t \leq\left\lceil\frac{H\left(Q, i_{2}\right)-3 k-2}{3}\right\rceil\right\}$ is an independent dominating set of $G-v$ of size $i(G)$, and so, $i(G) \geq i(G-v)$. To prove the inverse inequality, let $T$ be an $i(G-v)$-set. As above, we have $\left|T \cap\left\{v_{i_{j}, s, a}, v_{i_{j}, s, b}\right\}\right| \geq 1$ for each $1 \leq j \leq r$ and $1 \leq s \leq q_{i_{j}}$, and $\left|T \cap\left\{v_{i_{1}+1}, v_{i_{1}+2}, \ldots, v_{i_{2}-1}\right\}\right| \geq \frac{H\left(Q, i_{1}\right)}{3}$. Furthermore, to dominate the vertices $v_{i_{j}+2}, \ldots, v_{i_{j+1}-1}$, we 
must have $\left|T \cap\left\{v_{i_{j}+2}, \ldots, v_{i_{j+1}-1}, v_{i_{j+1}}\right\}\right| \geq \frac{H\left(Q, i_{j}\right)}{3}$ for $3 \leq j \leq r$. Now, to dominate the vertices $v_{i_{2}+1}, \ldots, v_{i_{2}+3 k+1}$, we must have $\left|T \cap\left\{v_{i_{2}}, \ldots, v_{i_{2}+3 k+1}\right\}\right| \geq k+1$, and to dominate the vertices $v_{i_{2}+3 k+3}, \ldots, v_{i_{3}-1}$, we must have $\left|T \cap\left\{v_{i_{2}+3 k+3}, \ldots, v_{i_{3}-1}\right\}\right| \geq \frac{H\left(Q, i_{2}\right)}{3}-k-1$. This implies that $|T| \geq i(G)$, and so, $i(G)=i(G-v)$.

Thus, $G$ is an independent domination stable graph, and the proof is complete.

The proof of the next result is straightforward and therefore omitted.

Proposition 13. If $G \in \mathcal{J}_{4}$, then $G$ is an independent domination stable graph.

In order to present our constructive characterization of independent domination stable unicyclic graphs, we define a family of graphs as follows. Let $\mathcal{G}$ be the family of graphs $G$ that can be obtained from a sequence $G_{1}, G_{2}, \ldots, G_{k}$ of graphs for some $k \geq 1$, where $G_{1} \in \mathcal{J}-\mathcal{T}$ if $k=1$ and $G_{1} \in \mathcal{J}$ if $k \geq 2$, and $G=G_{k}$. If $k \geq 2, G_{i+1}$ can be obtained from $G_{i}$ by one of the following operations.

Operation $\mathcal{O}_{1}$ : If $u \in W\left(G_{i}\right)$, then $\mathcal{O}_{1}$ adds a spider $S_{1}$ with head $s$ and an edge $u s$ to obtain $G_{i+1}$ (see Figure 3).

Operation $\mathcal{O}_{2}:$ If $u \in V\left(G_{i}\right)$, then $\mathcal{O}_{2}$ adds a spider $S_{q}(q \geq 2)$ with head $s$ and an edge $u s$ to obtain $G_{i+1}$ (see Figure 3).

Operation $\mathcal{O}_{3}$ : If $G_{i}$ is a tree and $(x, y) \in W^{1,1}\left(G_{i}\right)$, then $\mathcal{O}_{3}$ adds a graph $H_{k_{1}, k_{2}}\left(k_{1}=k_{2}=0\right)$ and edges $a x$, by to obtain $G_{i+1}$ (see Figure 1).

Operation $\mathcal{O}_{4}$ : If $G_{i}$ is a tree, $x \in W\left(G_{i}\right)$ and $y \in V\left(G_{i}\right)$, then $\mathcal{O}_{4}$ adds a graph $H_{k_{1}, k_{2}}\left(k_{1}=0, k_{2} \geq 1\right)$ and edges $a x$, by to obtain $G_{i+1}$ (see Figure 1).

Operation $\mathcal{O}_{5}$ : If $G_{i}$ is a tree, $x, y \in V\left(G_{i}\right)$, then $\mathcal{O}_{5}$ adds a graph $H_{k_{1}, k_{2}}\left(k_{1} \geq 1, k_{2} \geq 1\right)$ and edges $a x$, by to obtain $G_{i+1}$ (see Figure 1).

Operation $\mathcal{O}_{6}$ : If $G_{i}$ is a tree, $x, y \in V\left(G_{i}\right)$, then $\mathcal{O}_{6}$ adds a graph $P\left(6,\left(0,0, k_{1}, k_{2}, 0,0\right)\right)\left(k_{1} \geq 0, k_{2} \geq 0\right)$ and edges $v_{3} x, v_{4} y$ to obtain $G_{i+1}$ (see Figure 4).

Theorem 4. Let $G \in \mathcal{G}$ be a graph of order $n \geq 3$. Then, $G$ is an independent domination stable graph.

Proof. Suppose that $G \in \mathcal{G}$. Then, there exists a sequence of graphs $G_{1}, G_{2}, \ldots, G_{k}(k \geq 1)$ such that $G_{1} \in \mathcal{J}-\mathcal{T}$ if $k=1$ and $G_{1} \in \mathcal{J}$ if $k \geq 2$, and if $k \geq 2$, then $G_{i+1}$ can be obtained from $G_{i}$ by one of the operations $\mathcal{O}_{1}, \mathcal{O}_{2}, \cdots, \mathcal{O}_{6}$. We proceed by induction on the number of operations used to construct $G$. If $k=1$, the result holds by Propositions 11,12, and 13. Assume that the result holds for each graph $G \in \mathcal{G}$, which can be obtained from a sequence of operations of length $k-1$, and let $G^{\prime}=G_{k-1}$. By the induction hypothesis, $G^{\prime}$ is an independent domination stable graph. Since $G=G_{k}$ is obtained by one of the operations $\mathcal{O}_{1}, \mathcal{O}_{2}, \cdots, \mathcal{O}_{6}$ from $G^{\prime}$, we conclude from Propositions 5, 7, and 9 that $G$ is an independent domination stable unicyclic graph.

Theorem 5. Let $G$ be a unicyclic graph of order $n \geq 3$. Then, $G$ is an ID-stable graph if and only if $G \in \mathcal{G}$.

Proof. According to Theorem 4, we need only to prove necessity. Let $G$ be an ID-stable unicyclic graph of order $n \geq 3$. The proof is by induction on $n$. Let $n \geq 11$, and let the statement hold for all ID-stable unicyclic graphs of order less than $n$. Assume that $G$ is an ID-stable unicyclic graph of order $n$. Let $C=\left(v_{1} v_{2} \ldots v_{p}\right)$ be the unique cycle of $G$. If $G$ is a cycle, then $p=n$, and Proposition 1 implies that $G \in \mathcal{J}_{3} \subseteq \mathcal{G}$. Now, we consider the case $p<n$. Choose a vertex $u \in V(G)-V(C)$ such that the distance between the vertex $u$ and the set $V(C)$ is as large as possible. Assume that $v_{1} u_{1} u_{2} \cdots u_{\ell} u$ is the shortest $(u, V(C))$-path. If $\ell \geq 2$, then similar to the proof of Theorem 2, $G$ can be obtained from $G_{k-1}$ by one of the operations $\mathcal{O}_{1}$ or $\mathcal{O}_{2}$, and so, $G \in \mathcal{G}$. Assume that $\ell \leq 1$.

First, assume $v_{i}$ is not a support vertex for each $i \in\{1, \ldots, p\}$. Then, $G=C(n, Q)$ for some $Q \in \mathcal{N}^{n}$. If $D(Q)=0$, then it follows from Corollary 1 that $G \in \mathcal{J}_{1}$. If $D(Q)=1$, then it follows from Theorem 3 that $G \in \mathcal{J}_{2}$. If $D(Q) \geq 2$, then we conclude from Propositions 10 and 12 that $G \in \mathcal{J}_{3}$. 
Now, suppose that $v_{i}$ is a support vertex for some $i \in\{1, \ldots, p\}$, say $i=2$. Assume $c$ is a leaf adjacent to $v_{2}$. We conclude from Propositions 2 and 3 that $v_{2}$ is not a strong support vertex and is not adjacent to a support vertex. It follows that $d_{G}\left(v_{2}\right)=3$ and that $v_{1}, v_{3}$ are not support vertices. Let $k_{1}$ be the number of pendant paths of length two beginning at $v_{1}$ and $k_{2}$ be the number of pendant paths of length two beginning at $v_{3}$. Let $G^{\prime}$ be the graph obtained from $G$ by removing $v_{1}, v_{2}, v_{3}$ and the vertices of all pendant paths at $v_{1}, v_{3}$. By Proposition 6 , we have $i(G)=i\left(G^{\prime}\right)+k_{1}+k_{2}+1$. If $G^{\prime}$ is not an ID-stable graph, then $i\left(G^{\prime}-v\right) \neq i\left(G^{\prime}\right)$ for some vertex $v \in V\left(G^{\prime}\right)$, and it follows from Proposition 6 that $i(G-v)=i\left(G^{\prime}-v\right)+k_{1}+k_{2}+1 \neq i\left(G^{\prime}\right)+k_{1}+k_{2}+1=i(G)$, which is a contradiction. Hence, $G^{\prime}$ is an ID-stable graph, and by the induction hypothesis, we have $G^{\prime} \in \mathcal{G}$. If $k_{1} \geq 1, k_{2} \geq 1$, then $T$ can be obtained from $G^{\prime}$ by operation $\mathcal{O}_{5}$, and so, $G \in \mathcal{G}$. Assume that $k_{1}=k_{2}=0$. Then, we have $d_{G}\left(v_{1}\right)=d_{G}\left(v_{3}\right)=2$. Let $S$ be a $i\left(G-v_{2}\right)$-set. Since $G$ is an ID-stable graph, we have $i(G)=i\left(G-v_{2}\right)$. To dominate the vertices $c, v_{1}, v_{3}$, we must have $c \in S,\left|s \cap\left\{v_{1}, v_{n}\right\}\right| \geq 1$ and $\left|s \cap\left\{v_{3}, v_{4}\right\}\right| \geq 1$. Suppose, without loss of generality, that $v_{4}, v_{n} \in S$. Then, $S-\{c\}$ is an $i\left(G^{\prime}\right)$-set containing $v_{4}, v_{n}$, and so, $\left(v_{3}, v_{n}\right) \in W^{1,1}$. Now, $T$ can be obtained from $G^{\prime}$ by operation $\mathcal{O}_{3}$, and so, $G \in \mathcal{G}$. Finally, let $k_{1}=0$ and $k_{2} \geq 1$. As above, we can see that $v_{4} \in W\left(G^{\prime}\right)$, and since $T$ can be obtained from $G^{\prime}$ by operation $\mathcal{O}_{4}$, we have $T \in \mathcal{G}$. This completes the proof.

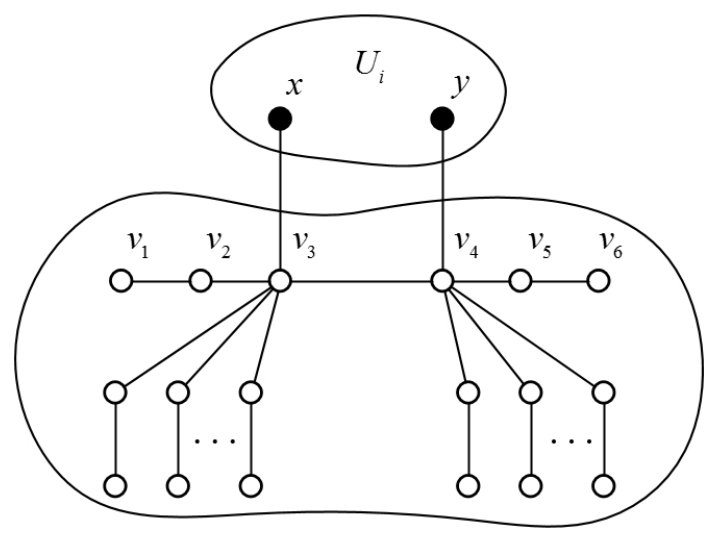

Figure 4. The operation $\mathcal{O}_{6}$.

\section{Bounds}

In this section, we provide sharp bounds on ID-stable trees. First, we present a lower bound and characterize all extremal trees. Let $\mathfrak{T}_{1}$ be the family of trees $T$ that can be obtained from a sequence $T_{1}$, $T_{2}, \ldots, T_{k}$ of trees for some $k \geq 1$, where $T_{1}$ is $P_{2}$ and $T=T_{k}$. If $k \geq 2$, then all but at most one of $T_{i+1}$ can be obtained from $T_{i}$ by operation $\mathcal{T}_{1}$, and that one (if any) can be obtained from $T_{i}$ by operation $\mathcal{T}_{2}$ for $q=2$.

Theorem 6. Let $T$ be an ID-stable tree of order $n \geq 2$. Then:

$$
i(T) \geq\left\lceil\frac{n}{3}\right\rceil
$$

with equality if and only if $T \in \mathfrak{T}_{1}$.

Proof. By Theorem 2, we have $T \in \mathcal{T}$. Thus, there exists a sequence of trees $T_{1}, T_{2}, \ldots, T_{k}(k \geq 1)$ such that $T_{1}$ is $P_{2}$, and if $k \geq 2$, then $T_{i+1}$ can be obtained from $T_{i}$ by one of the operations $\mathcal{T}_{1}$ or $\mathcal{T}_{2}$. We proceed by induction on the number of operations used to construct $T$. If $k=1$, the result is trivial. 
Assume the result holds for each tree $T \in \mathcal{T}$, which can be obtained from a sequence of operations of length $k-1$, and let $T^{\prime}=T_{k-1}$. By Proposition 4 and the induction hypothesis, we obtain:

$$
i(T)=i\left(T^{\prime}\right)+i\left(S_{q}\right) \geq\left\lceil\frac{n-2 q-1}{3}\right\rceil+\left\lceil\frac{2 q+1}{3}\right\rceil \geq\left\lceil\frac{n}{3}\right\rceil .
$$

The equality holds if and only if $i\left(T^{\prime}\right)=\left\lceil\frac{n-2 q-1}{3}\right\rceil$ and $i\left(S_{q}\right)=\left\lceil\frac{2 q+1}{3}\right\rceil$. It follows from the induction hypothesis that $T^{\prime} \in \mathfrak{T}_{1}$. Furthermore, we deduce from $i\left(S_{q}\right)=\left\lceil\frac{2 q+1}{3}\right\rceil$ that $q \leq 3$. First, let $q=3$. It follows from Equation (1) that:

$$
\left\lceil\frac{n-7}{3}\right\rceil+\left\lceil\frac{7}{3}\right\rceil=\left\lceil\frac{n}{3}\right\rceil
$$

yielding $n \equiv 1(\bmod 3)$. This implies that $3 \mid n\left(T^{\prime}\right)$, which is a contradiction by construction of trees in $\mathfrak{T}_{1}$. Hence, $q \leq 2$. If $T_{i+1}$ is obtained from $T_{i}$ by operation $\mathcal{T}_{1}$ for each $2 \leq i \leq k-1$, then clearly, $T \in \mathcal{T}_{1}$. Assume that one of the $T_{i+1}$ 's is obtained from $T_{i}$ by operation $\mathcal{T}_{2}$ for $q=2$. Then, clearly, $n\left(T^{\prime}\right)=n-2 q-1=3(k-1)+1$. If $q=2$, then $n(T)=3(k-1)+6$, and we have $\left\lceil\frac{n-2 q-1}{3}\right\rceil+\left\lceil\frac{2 q+1}{3}\right\rceil=k+2>k+1=\left\lceil\frac{n(T)}{3}\right\rceil$, which is a contradiction. Thus, $q=1$, and this implies that $T \in \mathfrak{T}_{1}$.

Let $\mathcal{F}_{1}$ be the family of all spiders $S_{q}$ for $q \geq 2, \mathcal{F}_{2}$ be the family of trees obtained from two spiders $S_{p}$ and $S_{q}$ by joining their heads, $\mathcal{F}_{3}$ be the family of trees obtained from two spiders $S_{p}$ and $S_{q}$ by joining the head of $S_{p}$ to a knee of $S_{q}$, and $\mathcal{F}_{4}$ be the family of trees obtained from two spiders $S_{p}$ and $S_{q}$ by joining the head of $S_{p}$ to a foot of $S_{q}$ where $p \geq q=2$ or $p, q \geq 3$. For example, the trees obtained by $\mathcal{F}_{2}, \mathcal{F}_{3}$, and $\mathcal{F}_{4}$ when $p=q=3$ are illustrated in Figure 5 .

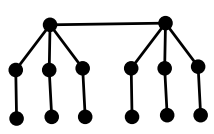

(a)

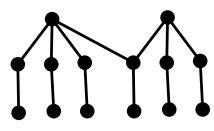

(b)

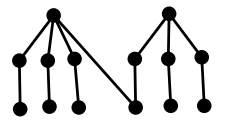

(c)

Figure 5. (a) Tree $\mathcal{F}_{2} ;(\mathbf{b})$ tree $\mathcal{F}_{3} ;(\mathbf{c})$ tree $\mathcal{F}_{4}$.

The next result is an immediate consequence of Proposition 4.

Observation 1. If $T \in \cup_{i=1}^{4} \mathcal{F}_{i}$, then $i(T)=\left\lceil\frac{n-2}{2}\right\rceil$.

Theorem 7. Let $T$ be an ID-stable tree of order $n \geq 5$. Then:

$$
i(T) \leq\left\lceil\frac{n-2}{2}\right\rceil
$$

with equality if and only if $T \in \cup_{i=1}^{4} \mathcal{F}_{i}$.

Proof. The proof is by induction on $n$. If $n=5$, then by Propositions 2 and 3 , we have $T=P_{5}$, and the result holds. Let $n \geq 6$, and let the statement hold for all ID-stable trees of order less than $n$. Assume that $T$ is an ID-stable tree of order $n$. By Propositions 2 and 3, we deduce that $\operatorname{diam}(T) \geq 4$. If $\operatorname{diam}(T)=4$, then by Propositions 2 and 3, $T$ is the healthy spider $\operatorname{Spider}\left(d_{T}\left(v_{3}\right)\right)$, and so, $i(T)=d_{T}\left(v_{3}\right)=\left\lceil\frac{n(T)-2}{2}\right\rceil$ and $T \in \mathcal{F}_{1}$. Suppose that $\operatorname{diam}(T) \geq 5$. Let $v_{1} v_{2} \ldots v_{k}(k \geq 5)$ be a diametrical path in $T$ such that $d_{T}\left(v_{3}\right)$ is as large as possible and root $T$ at $v_{k}$. By Propositions 2 and 3, we have $d_{T}\left(v_{2}\right)=2$ and that $v_{3}$ is not a support vertex. Hence, $T_{v_{3}}=S_{d_{T}\left(v_{3}\right)-1}$. Assume that 
$p=d_{T}\left(v_{3}\right)-1$. Let $T^{\prime}=T-T_{v_{3}}$. Since $T$ is an ID-stable tree, we deduce from Proposition 4 that for any vertex $v \in V\left(T^{\prime}\right)$,

$$
i\left(T^{\prime}-v\right)+p=i(T-v)=i(T)=i\left(T^{\prime}\right)+p
$$

and this implies that $i\left(T^{\prime}-v\right)=i\left(T^{\prime}\right)$. Hence, $T^{\prime}$ is an ID-stable tree. It follows from the induction hypothesis that $i\left(T^{\prime}\right) \leq\left\lceil\frac{n-2 p-3}{2}\right\rceil$, and hence,

$$
\begin{aligned}
i(T) & \leq i\left(T^{\prime}\right)+i\left(S_{p}\right) \\
& \leq\left\lceil\frac{n-2 p-3}{2}\right\rceil+p \\
& =\left\lceil\frac{n-3}{2}\right\rceil \\
& \leq\left\lceil\frac{n-2}{2}\right\rceil .
\end{aligned}
$$

The equality holds if and only if $\operatorname{diam}(T)=4$ or $\operatorname{diam}(T) \geq 5$ and $i\left(T^{\prime}\right)=\left\lceil\frac{n\left(T^{\prime}\right)-2}{2}\right\rceil=\left\lceil\frac{n-2 p-3}{2}\right\rceil$ and $n$ is even, and this if and only if $T \in \mathcal{F}_{1}$ or $\operatorname{diam}(T) \geq 5$ and $T^{\prime} \in \mathcal{F}_{1}$ by the induction hypothesis. Thus, the equality holds if and only if $T \in \cup_{i=1}^{4} \mathcal{F}_{i}$, and the proof is complete.

\section{Conclusions}

In this note, we studied the ID-stable graphs. Some basic properties of ID-stable graphs were presented and new independent domination stable graphs constructed from an old one. We also characterized all independent domination stable trees and unicyclic graphs. In addition, we proved that for any tree $T$ of order $n \geq 5,\left\lceil\frac{n}{3}\right\rceil \leq i(T) \leq\left\lceil\frac{n-2}{2}\right\rceil$, and we characterized all trees attaining the lower and upper bound. An interesting problem is to find sharp lower and upper bounds on the independent domination number of ID-stable graphs. The other problem is to characterize all ID-stable bicyclic graphs. Another problem is to study algorithm running times to decide independent domination graphs.

Author Contributions: Z.S. and S.M.S. contributed to the supervision, methodology, validation, project administration, and formal analysis. P.W., H.J., S.N.-M., and L.V. contributed to the investigation, resources, and some computations and wrote the initial draft of the paper, which was investigated and approved by Z.S. S.M.S. wrote the final draft.

Funding: This work is supported by the Natural Science Foundation of Guangdong Province under Grant 2018A0303130115, the Science and Technology Program of Guangzhou (No. 201904010493), and the Specialized Fund for Science and Technology Platform and Talent Team Project of Guizhou Province (No. QianKeHePingTaiRenCai [2016]5609).

Conflicts of Interest: The authors declare no conflict of interest.

\section{Appendix A}

\section{Appendix A.1. Trees}

By applying the constructive method as above, we obtain all ID-stable trees with order up to 12 , and the statistics of the number of trees with different orders is presented in Table A1.

We list all the independent domination stable trees with orders from 5 to 12 in Figure A1.

\section{Appendix A.2. Unicyclic Graphs}

By applying the constructive method as above, we obtain all independent domination stable unicyclic graphs with order from 3 to 10, and the statistics of the number of unicyclic graphs with different orders is presented in Table A2. 
We here list all the independent domination stable unicyclic graphs with orders from 3 to 10 in Figure A2.
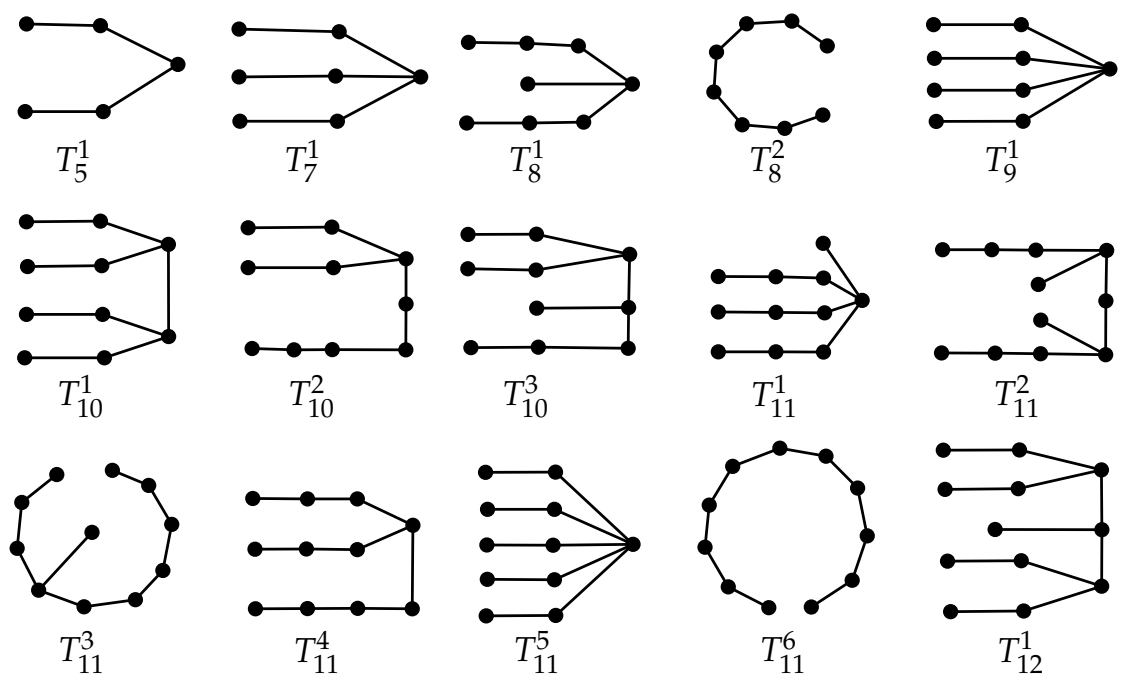

$$
T_{11}^{3}
$$

$T_{11}^{4} \quad T_{11}^{5}$

$T_{11}^{6}$

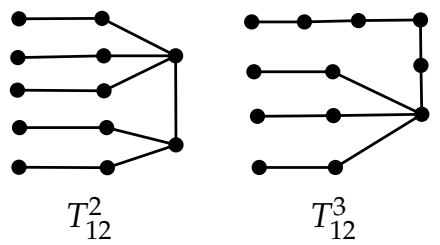

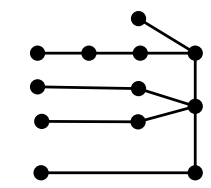

$T_{12}^{4}$

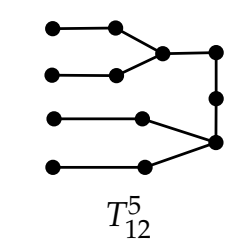

Figure A1. Independent domination stable trees with orders from 5 to 12.
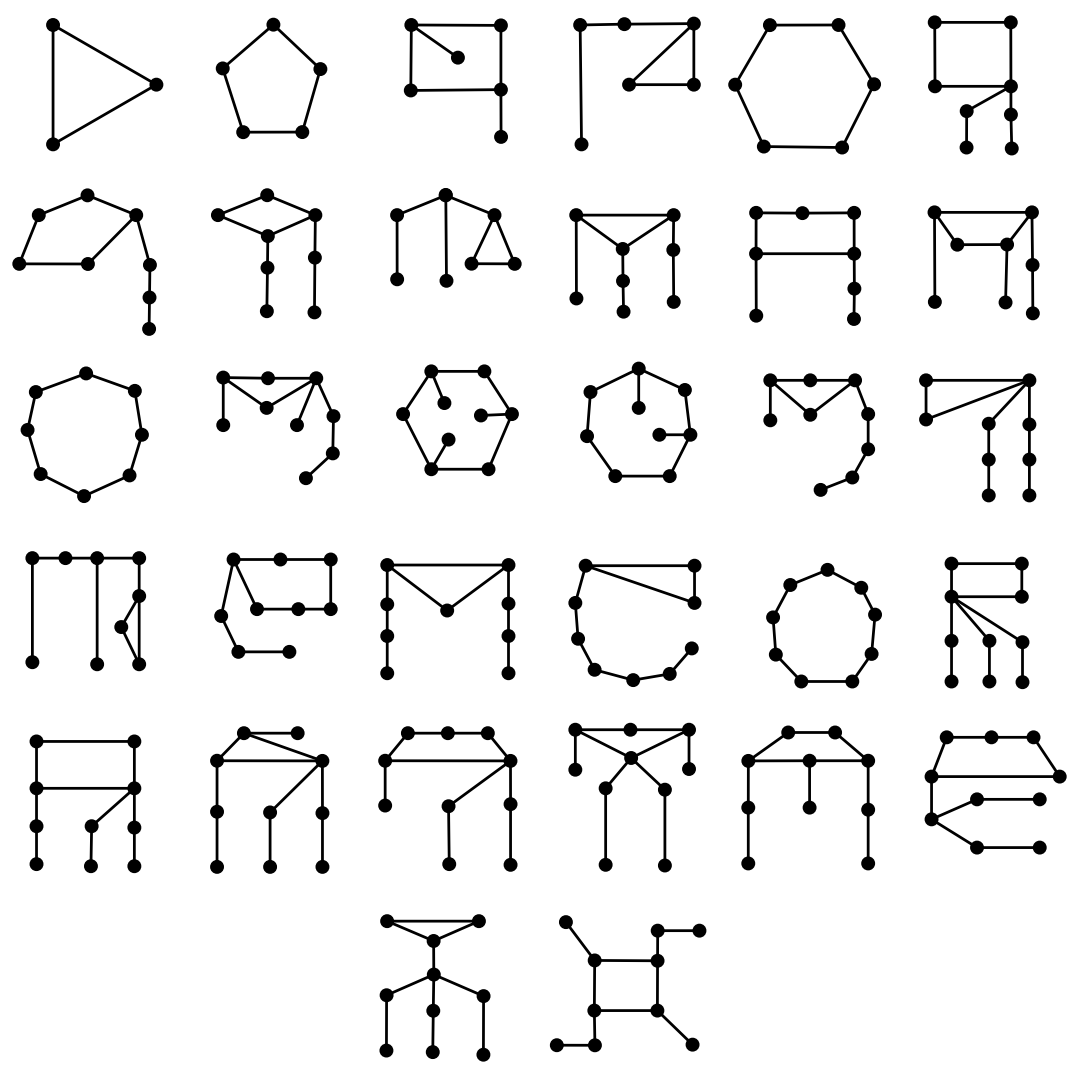

Figure A2. All independent domination stable unicyclic graphs of orders from 3 to 10. 
Table A1. The number of independent domination stable trees with different orders.

\begin{tabular}{llllllllllll}
\hline Order & 2 & 3 & 4 & 5 & 6 & 7 & 8 & 9 & 10 & 11 & 12 \\
Number & 1 & 0 & 0 & 1 & 0 & 1 & 2 & 1 & 3 & 4 & 5 \\
\hline
\end{tabular}

Table A2. The number of independent domination stable unicyclic graphs with different orders.

\begin{tabular}{lllllllll}
\hline Order & 3 & 4 & 5 & 6 & 7 & 8 & 9 & 10 \\
Number & 1 & 0 & 1 & 3 & 0 & 8 & 10 & 9 \\
\hline
\end{tabular}

\section{References}

1. Goddard, W.; Henning, M.A. Independent domination in graphs: A survey and recent results. Discret. Math. 2013, 313, 839-854. [CrossRef]

2. Desormeaux, W.J.; Haynes, T.W.; Henning, M.A. Total domination stable graphs upon edge addition. Discret. Math. 2010, 310, 3446-3454. [CrossRef]

3. Zhu, E.; Liu, C.; Deng, F.; Rao, Y. On upper total domination versus upper domination in graphs. Graphs Comb. 2019, 35, 767-778. [CrossRef]

4. Cockayne, E.J.; Dreyer, P.A., Jr.; Hedetniemi, S.M.; Hedetniemi, S.T. Roman domination in graphs. Discret. Math. 2004, 278, 11-22. [CrossRef]

5. Zhu, E.; Shao, Z. Extremal problems on weak Roman domination number. Inf. Process. Lett. 2018, 138, 12-18. [CrossRef]

6. Zhu, E.; Shao, Z.; Xu, J. Semitotal domination in claw-free cubic graphs. Graphs Comb. 2017, 33, 1119-1130. [CrossRef]

7. Zhu, E.; Liu, C. On the semitotal domination number of line graphs. Discret. Appl. Math. 2019, 254, $295-298$. [CrossRef]

8. Haynes, T.W.; Hedetniemi, S.T.; Slater, P.J. Fundamentals of Domination in Graphs; CRC Press: Boca Raton, FL, USA, 1998.

9. Haynes, T.W.; Hedetniemi, S.; Slater, P. Domination in Graphs: Volume 2: Advanced Topics; CRC Press: Boca Raton, FL, USA, 1998.

10. Ao, S. Independent Domination Critical Graphs. Ph.D. Thesis, University of Victoria, Victoria, BC, Canada, 1994.

11. Edwards, M. Vertex-Criticality and Bicriticality for Independent Domination and Total Domination in Graphs. Ph.D. Thesis, University of Victoria, Victoria, BC, Canada, 2015.

12. Edwards, M.; Finbow, A.; MacGillivray, G.; Nasserasr, S. Independent domination bicritical graphs. Australas. J. Comb. 2018, 72, 446-471.

13. Edwards, M.; MacGillivray, G. The diameter of total domination and independent domination vertex-critical graphs. Australas. J. Comb. 2012, 52, 33-40.

14. Ananchuen, N.; Ruangthampisan, S.; Ananchuen, W.; Caccetta, L. On minimum cutsets in independent domination vertex-critical graphs. Australas. J. Comb. 2018, 71, 369-380.

15. Chellali, M.; Haynes, T.W. Double domination stable graphs upon edge removal. Australas. J. Comb. 2010, $47,157-164$.

16. Desormeaux, W.J.; Haynes, T.W.; Henning, M.A. An extremal problem for total domination stable graphs upon edge removal. Discret. Appl. Math. 2011, 159, 1048-1052. [CrossRef]

17. Desormeaux, W.J.; Haynes, T.W.; Henning, M.A. Total domination changing and stable graphs upon vertex removal. Discret. Appl. Math. 2011, 159, 1548-1554. [CrossRef] 